\title{
Tropomyosin is required for cardiac morphogenesis, myofibril assembly, and formation of adherens junctions in the developing mouse embryo
}

\author{
Caroline R. McKeown, Roberta B. Nowak, David S. Gokhin, and Velia M. Fowler \\ Department of Cell and Molecular Biology, The Scripps Research Institute, La Jolla, CA 92037
}

\section{Abstract}

Background-We explored a function for tropomyosin (TM) in mammalian myofibril assembly and cardiac development by analyzing a deletion in the mouse TPM1 gene targeting aTM1, the major striated muscle TM isoform.

\begin{abstract}
Results-Mice lacking aTM1 are embryonic lethal at E9.5 with enlarged, misshapen, and nonbeating hearts characterized by an abnormally thin myocardium and reduced trabeculae. aTM1deficient cardiomyocytes do not assemble striated myofibrils, instead displaying aberrant nonstriated F-actin fibrils with a-actinin puncta dispersed irregularly along their lengths. aTM1's binding partner, tropomodulin1 (Tmod1), is also disorganized, and both myomesin-containing thick filaments as well as titin Z1Z2 fail to assemble in a striated pattern. Adherens junctions are reduced in size in aTM1-deficient cardiomyocytes, $a$-actinin/F-actin adherens belts fail to assemble at apical cell-cell contacts, and cell contours are highly irregular, resulting in abnormal cell shapes and a highly folded cardiac surface. In addition, Tmod1-deficient cardiomyocytes exhibit failure of $\mathrm{a}$-actinin/F-actin adherens belt assembly.
\end{abstract}

Conclusions-Absence of aTM1 resulting in unstable F-actin may preclude sarcomere formation and/or lead to degeneration of partially assembled sarcomeres due to unregulated actomyosin interactions. Our data also identify a novel aTM1/Tmod1-based pathway stabilizing F-actin at cell-cell junctions, which may be required for maintenance of cell shapes during embryonic cardiac morphogenesis.

\section{Keywords}

a-actinin; cell junction; cardiomyocyte; heart; myofibrillogenesis; myomesin; thin filament; titin; tropomodulin 1

\section{Introduction}

Vertebrate cardiac morphogenesis involves a complex developmental program of cardiacspecific gene expression, cell proliferation, migration, and shape changes, as well as

\footnotetext{
*Author and address for correspondence: Velia M. Fowler, Ph.D., Department of Cell and Molecular Biology, The Scripps Research Institute, 10550 N Torrey Pines Road, CB163, La Jolla, CA 92037, Phone: 858-784-8277, Fax: 858-784-8753, velia@ @cripps.edu. 
assembly of striated myofibrils (Gregorio and Antin, 2000; Auman and Yelon, 2004; Olson, 2006). Myofibril assembly and contractile function are believed to control cardiac cell shape and interactions during morphogenesis of the heart tube and chamber formation (Manasek, 1981). Cardiac myofibrils assemble at the cell membrane, beginning as fibrillar bundles of actin filaments with closely spaced Z-bodies (i.e., puncta of a-actinin), maturing into sarcomeres with regularly spaced Z-lines (striated a-actinin), where myosin (thick) filaments act as the contractile motor and actin (thin) filaments act as the scaffold (Clark et al., 2002). Thin filaments are stabilized by both lateral interactions and end-capping; tropomyosin (TM)-troponin complexes associate along the lengths of thin filaments, which are capped at their barbed ends by the capping protein CapZ in the Z-line and at their pointed ends by tropomodulin1 (Tmod1) at the H-zone periphery (Clark et al., 2002). Hundreds of mutations in genes encoding thin filament-associated proteins, such as actin, aactinin, nebulette, troponins I and T, and TM-result in diverse congenital cardiomyopathies in humans (Szczesna-Cordary et al., 2012). In particular, mutations in TM that affect regulation of actomyosin contraction result in dilated and hypertrophic cardiomyopathy (Michele and Metzger, 2000). However, any putative involvement of TM in mammalian myofibril assembly or in vivo cardiac development remains unclear.

Gene perturbation experiments in Drosophila, zebrafish, and mice have provided insights into the roles of several thin filament components in de novo myofibril assembly during striated muscle development. Troponin I is essential for myofibril assembly in Drosophila indirect flight muscle (IFM); in troponin I-deficient IFMs, developing muscle fibers assemble clumps and fibrils of disorganized thin and thick filaments with expanded Z-line structures, but no organized sarcomeres are observed (Nongthomba et al., 2004). This phenotype is partly (but not completely) due to unregulated actomyosin interactions in absence of troponin I, since partial rescue of sarcomere assembly is observed upon genetic deletion of myosin force-producing heads (Nongthomba et al., 2004). Troponin $\mathrm{T}$ is essential for myofibril assembly and development of the mouse heart, where deletion of troponin $\mathrm{T}$ causes failure of myofibril assembly, severe cardiac dilation, and lack of heartbeat, leading to embryonic lethality at Ê10 (Nishii et al., 2008). Similar observations demonstrating essential functions for troponins in sarcomere formation, as well as exacerbation of defects by unregulated actomyosin activity during myofibril assembly, have been made in studies of zebrafish striated muscles (Sehnert et al., 2002; Huang et al., 2009; Ferrante et al., 2011). The primary direct evidence for a role of TM in myofibril assembly comes from the Mexican axolotl, in which the naturally occurring cardiac mutation eliminates TM expression and disrupts cardiac myofibril assembly via aberrant intracellular targeting of a host of thin filament and sarcomere-associated proteins and lack of coalescence of organized sarcomeres (Lemanski, 1973; Lemanski, 1979; Lemanski et al., 1980; Fuldner et al., 1984; Starr et al., 1989; Erginel-Unaltuna and Lemanski, 1994; La France and Lemanski, 1994; Zajdel et al., 1998; Zajdel et al., 1999; McLean et al., 2006; Zajdel et al., 2007). A heart-specific TM isoform (tpm4) is also essential for myofibril assembly and heartbeat in zebrafish (Zhao et al., 2008), but the lack of confirmed association of tpm4 with thin filaments makes it unclear whether the myofibril assembly defects observed in tpm4-deficient zebrafish represent primary or indirect events. Regardless, to date, no experimental perturbations of TM have been performed during de 
novo myofibril assembly in the developing mammalian heart, and the role of TM in mammalian myofibril assembly, distinct from its role in the regulation of muscle contraction in mature myofibrils in the adult heart, remains incompletely understood.

Mammals have four TM genes that encode $>40$ TM protein isoforms (Gunning et al., 2008). The principal TM in mammalian striated muscles, including the embryonic heart, is aTM1, a product of the TPM1 gene (Muthuchamy et al., 1993; Schleef et al., 1993). aTM1 is an ahelical protein that forms parallel coiled-coil dimers that associate in a head-to-tail fashion and bind along the sides of thin filaments. In its contractile regulatory function, aTM1 binds to actin and troponin $\mathrm{T}$ in the troponin complex to mediate $\mathrm{Ca}^{2+}$-regulated actomyosin interactions. The aTM1-troponin complex inhibits the actomyosin interaction in its resting state; upon $\mathrm{Ca}^{2+}$ binding to troponin $\mathrm{C}$, this inhibition is released, allowing for muscle contraction (Kobayashi and Solaro, 2005). In addition to its contractile regulatory function, aTM1 inhibits F-actin binding of sarcomeric a-actinin (as well as other F-actin binding proteins), thereby establishing domains along F-actin (Goll et al., 1972; Stromer and Goll, 1972; Maruyama and Ohashi, 1978; Zeece et al., 1979; Burgess et al., 1987; Blanchoin et al., 2001). TM also stabilizes F-actin from depolymerization and severing in vitro; both nonmuscle TM- and aTM1-coated actin filaments depolymerize from their pointed ends more slowly than bare F-actin (Broschat et al., 1989; Broschat, 1990; Weber et al., 1994; Weber et al., 1999). Moreover, TM blocks both the actin-depolymerizing activity of ADF/ cofilin (Bernstein and Bamburg, 1982; Nishida et al., 1984; Ono and Ono, 2002) and the actin-severing activity of gelsolin (Ishikawa et al., 1989). Due to its head-to-tail association, binding of the terminal aTM1 molecule at the pointed end is considerably weaker than binding of adjacent a TM1 subunits to actin along the aTM1 polymer (Wegner, 1979; Yang et al., 1979).

At the pointed ends of cardiac muscle thin filaments, the N-terminus of aTM1 interacts with Tmod1, an actin pointed-end capping protein that stabilizes F-actin and regulates F-actin lengths (Gregorio et al., 1995; Littlefield et al., 2001; Mudry et al., 2003; Moyer et al., 2010; Gokhin and Fowler, 2011; Yamashiro et al., 2012). Tmod1 and aTM1 function synergistically, whereby aTM1 greatly enhances the ability of Tmod1 to cap actin filament pointed ends, while Tmod1 enhances the ability of aTM1 to stabilize F-actin and prevent pointed-end depolymerization (Weber et al., 1994; Weber et al., 1999; Mudry et al., 2003; Kostyukova and Hitchcock-DeGregori, 2004). Deletion of Tmod1 is embryonic lethal, with Tmod1 ${ }^{-/}$embryos exhibiting normal morphology through E7.5 but showing defects in cardiac looping morphogenesis, chamber formation, and myofibril assembly by E8.5, resulting in aborted establishment of the circulation and death by E9.5-10.5 (Chu et al., 2003; Fritz-Six et al., 2003; McKeown et al., 2008). Tmod1 ${ }^{-/-}$cardiomyocytes display abnormal bundles of F-actin and $\mathrm{a}$-actinin, as well as occasional premyofibril-like structures with closely spaced $\mathrm{a}$-actinin puncta along continuous F-actin fibrils, but these structures fail to mature into striated myofibrils with regulated thin filament lengths (Fritz-Six et al., 2003). This may be due to lack of Tmod1-aTM1 interactions and destabilization of F-actin, but whether aTM1 assembles into these structures was not determined. However, Tmod $1^{-/-}$ hearts exhibit relatively normal thick filament assembly in association with some F-actin 
fibrils, as evidenced by normal striated organization of myomesin, a crosslinker of thick filaments in the M-line (Fritz-Six et al., 2003).

We set out to decipher the role of aTM1 in mammalian myofibril assembly by studying mice lacking aTM1, the major cardiac TM isoform, during embryonic development (Rethinasamy et al., 1998). We discovered that aTM1 is required for both cardiac development and myofibril assembly. Specifically, we demonstrate that hearts lacking aTM1 fail to form striated myofibrils. Instead, aTM1-deficient cardiomyocytes assemble aberrant F-actin fibrils associated with variably spaced sarcomeric $\mathrm{a}$-actinin puncta and larger a-actinin rod-like structures along their lengths. Tmod1 fails to assemble on the Factin fibrils in the absence of aTM1, and aTM1 fails to assemble into continuous F-actin fibrils in absence of Tmod1, suggesting that F-actin stabilization and length regulation to form sarcomeres requires cooperation between aTM1 and Tmod1. Moreover, the aberrant F-actin fibrils in a TM1 $1^{-/-}$hearts lack organized patterns of the titin $\mathrm{Z1Z2}$ domain or myomesin-containing thick filaments. This represents a more severe myofibril assembly defect than $\mathrm{Tmodl}^{-/-}$hearts (Fritz-Six et al., 2003), highlighting a novel interdependence of thin and thick filament assembly. Alternatively, absence of aTM1 may lead to degeneration of partially assembled sarcomeres via unregulated actomyosin interactions, as in troponin I and T mutants in flies, zebrafish, and mice (Huang et al., 1999; Sehnert et al., 2002; Nongthomba et al., 2004; Nishii et al., 2008; Ferrante et al., 2011). Moreover, we identify novel roles for aTM1 and Tmod1 in cardiomyocyte adherens belt assembly and cell adhesion, maintenance of cell shape, and regulation of heart size. The data presented here define several critical roles for aTM1 in mammalian cardiac morphogenesis and myofibril assembly, distinct from aTM1's better-characterized roles in regulation of muscle contraction in mature myofibrils.

\section{Results}

\section{Deletion of aTM1 results in embryonic lethality at E9.5 due to defects in cardiac morphogenesis and function}

A targeted deletion of exons $9 \mathrm{a}$ and $9 \mathrm{~b}$ from the mouse $T P M I$ gene was previously generated by two groups, using different strategies, resulting in a predicted null for the striated muscle aTM1 protein (Fig. 1A) (Blanchard et al., 1997; Rethinasamy et al., 1998). The a $T M 1^{+/-}$adults were phenotypically normal, with wild-type aTM1 protein levels in muscles and myofibrillar fractions despite a 50\% reduction in a TM1 mRNA (Blanchard et al., 1997; Rethinasamy et al., 1998). In contrast, the a TM1 ${ }^{-/}$mice were reported to be embryonic lethal sometime between E8 and E11.5 days of gestation, due to unknown causes (Blanchard et al., 1997; Rethinasamy et al., 1998). Hence, a TM1 ${ }^{-/}$mice were uncharacterized. We acquired the strain created by Rethinasamy and colleagues (Rethinasamy et al., 1998) and out-crossed it onto a C57B1/6J background to approximately 97\% congenicity. As expected, a TM1 ${ }^{+/-}$mice were completely viable and fertile (Table 1),

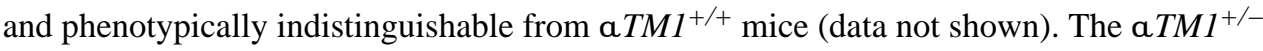
intercrosses failed to produce live a $T M 1^{-/-}$progeny, confirming that targeted deletion of aTM1 is embryonic lethal (Rethinasamy et al., 1998). 
To determine the lethal stage of the aTM1 ${ }^{-/-}$mice, we performed timed matings of aTM1 ${ }^{+/-}$mice and analyzed the resulting embryos at various stages of gestation. PCR genotyping showed that the aTM1 $1^{-/}$embryos were recovered at expected Mendelian ratios up to E9.5, but not at later stages (Table 1 and Fig. 1B). At E10.5, far fewer aTM1 ${ }^{-/-}$ embryos than expected were recovered, and the few recovered embryos were stunted, signifying that they were in process of resorption (Table 1). At subsequent times in development, we failed to find any a TM1 ${ }^{-/-}$embryos, suggesting that loss of aTM1 causes embryonic lethality between E9.5 and E10.5 (Table 1). To confirm that the aTM1 ${ }^{-/-}$allele was a true null allele, we performed Western blots on E9.5 embryonic lysates derived from the $\mathrm{aTMI}^{+/-}$intercrosses. Whereas a 36-37 $\mathrm{kD}$ aTM1 polypeptide was detected in both a TM1 $1^{+/+}$and $a T M 1^{+/-}$embryos, no aTM1 protein was detected in the a $T M 1^{-/-}$embryos (Fig. 1C), and no aTM1 staining was observed in whole-mount immunofluorescence of a $T M 1^{-/-}$hearts (data not shown). Interestingly, the a $T M 1^{+/-}$embryos consistently displayed reduced levels of aTM1 protein (Fig. 1C), suggesting that the compensatory mechanisms leading to wild-type levels of a TM1 protein in the adult hearts of $T M 1^{+/-}$mice (Rethinasamy et al., 1998) occur sometime later in development. Despite lower levels of aTM1 in aTM1+/- embryos (Fig. 1C), the gross morphology and development of the $\mathrm{aTM1}^{+/-}$embryos were indistinguishable from a $T M 1^{+/+}$embryos (data not shown).

The earliest developmental phenotype observed in the aTM1 ${ }^{-/-}$embryos is a defect in cardiac morphology and function. The a $T M 1^{-/-}$developing heart tube appeared enlarged and irregularly shaped as early as E8.5 (Fig. 2A-B, arrowheads). By E9.5, the enlargement of the aTM1-null heart was even more pronounced (Fig. 2C-D). In contrast to the aTM1 $1^{+/+}$ heart tube, which was smooth with a fairly consistent diameter (Fig. 2C), the E9.5 a TM1 ${ }^{-/-}$ heart tube appeared grossly misshapen with an enlarged ventricle and an abnormal constriction in the outflow tract (Fig. 2D, arrow). This constriction was particularly evident in the embryos that survived to a slightly later time in development (23 somite pairs; Fig. 3D), whose hearts additionally showed an excessive elongation of the inflow tract (Fig. 3C, D). The surface of the a $T M 1^{-/-}$heart tube also displayed wrinkles, which were more noticeable in later-stage embryos (Figs. 2D and 3B,C). The aTM1 ${ }^{-/-}$hearts continued to grow abnormally until the embryos were resorbed around E10.5 (Fig. 3). Moreover, the a TM1 $1^{-/}$embryonic hearts failed to contract; of 100 embryos collected between E8.5 and E9.5, 66 of the aTM1 $1^{+/+}$and aTM1 ${ }^{+/-}$embryos had beating hearts (66/72), but 0 of the aTM1 ${ }^{-/-}$embryonic hearts displayed contractions (0/28), as revealed by direct visual inspection during dissection. Normal cardiac function is required for vasculogenesis of the embryonic yolk sac during embryonic development (Lucitti et al., 2007; McKeown et al., 2008), and the $a T M 1^{+/+}$embryos had well-vascularized yolk sacs with large vessels containing blood cells (Fig. 2E), as expected. In contrast, the a $T M 1^{-/-}$yolk sacs failed to undergo embryonic vasculogenesis and appeared pale with no discernable blood vessels (Fig. 2F), consistent with a functional defect in cardiac muscle contraction. Thus, loss of aTM1 results in defects in cardiac morphogenesis and function during embryogenesis.

\section{Deletion of aTM1 results in abnormal cardiomyocyte morphology and organization}

To examine whether cardiomyocyte morphology and organization were affected by loss of aTM1, we performed histological analyses of sagittal sections of E9.5 hearts. In aTM1 ${ }^{+/+}$ 
hearts, the ventricular wall was organized into a compact myocardium, two cell layers thick, with cells closely apposed to one another and few gaps (Fig. 2G, $\mathrm{G}^{\prime}$, arrowhead). At this stage, the cardiomyocytes also began to form trabeculae extending from the compact myocardium into the cardiac jelly (Fig. 2G, Tr). However, in a $T M 1^{-/-}$hearts, the myocardium was uncompacted, instead appearing thinner and lacey, with frequent gaps between adjacent cells and between the two cell layers (Fig. $2 \mathrm{H}, \mathrm{H}^{\prime}$, open arrowheads). Moreover, the ventricular wall was only one cell layer thick in some regions of the aTM1-1heart (Fig. $2 \mathrm{H}^{\prime}$, black arrowhead). The E9.5 $\mathrm{aTM}^{-/-}$hearts also had fewer and wispier trabeculae. Comparison of a $T M 1^{+/+}$and $\mathrm{aTM1}^{-/-}$hearts at higher magnification using transmission electron microscopy (TEM) revealed that the a $T M 1^{-/-}$cardiomyocytes were often widely separated, with expanded intercellular spaces and abnormally long cellular extensions (Fig. 2I,J). Thus, cardiomyocyte organization and interactions are defective in the $\mathrm{aTM1}^{-/-}$heart.

Attenuated cell morphologies and expanded spaces between cells in the absence of aTM1 could account for the abnormally large hearts in the a $T M 1^{-/-}$embryos (Figs. 2D, 3).

Alternatively, the enlargement could reflect increased numbers of cells in the aTM1-/heart. To distinguish between these possibilities, we counted cells in whole mounts of the heart by two independent confocal microscopy approaches, using immunofluorescence staining for sarcomeric a-actinin as a marker for cardiomyocytes. First, we counted the number of dividing cardiomyocytes, as indicated by phospho-histone- $\mathrm{H} 3$ staining, and found that $\mathrm{aTM1^{+/+ }}$ and $\mathrm{aTM1}{ }^{-/-}$embryos had similar numbers of dividing nuclei at E9.5 (data not shown). Next, we also assayed cell number by counting the number of Hoechst-stained nuclei per defined region of interest in the embryonic heart. This analysis demonstrated that both $\mathrm{aTMI}^{+/+}$and $\mathrm{aTM1} 1^{-/-}$hearts had the same average number of nuclei $\left(\mathrm{a} T M 1^{+/+}=\right.$ $19.63 \pm 4.2$ and $\mathrm{aTMI}^{-/-}=19.67 \pm 5.8$ nuclei per $46 \mu \mathrm{m} \times 46 \mu \mathrm{m}$ area, $n=10$ areas from 2 embryos of each genotype). Thus, the overall number of cells and the proportion of dividing cells were unchanged in the a $T M 1^{-1-}$ embryonic hearts, indicating that the malformed larger heart was likely due to the attenuated cell shapes and abnormal intercellular gaps between the cardiomyocytes.

\section{Striated myofibrils fail to assemble and adherens junctions are dramatically smaller in aTM1-deficient cardiomyocytes}

aTM1 is a major component of the myofibril, localizing along the thin filaments in the sarcomere, stabilizing the thin filaments and playing a key role in thin filament regulation of muscle contraction (Clark et al., 2002; Kobayashi and Solaro, 2005). To examine myofibril structure and organization in a $T M 1^{-/-}$hearts, we performed TEM on E9.5 mouse embryos. In $\mathrm{aTM1}^{+/+}$and $\mathrm{aTM1} 1^{+/-}$embryos, cardiomyocytes displayed well-organized myofibrils characterized by repeating, $\sim 2-\mu \mathrm{m}$-long sarcomeres, with electron-dense Z-lines alternating with bundles of aligned thin and thick filaments (aTM1 ${ }^{+/-}$hearts shown in Fig. 4A, B). As expected from previous ultrastructural studies of cardiac morphogenesis (Manasek, 1968; Markwald, 1973; Chacko, 1976; Navaratnam et al., 1986), I-bands were not evident in myofibrils at this stage of cardiac development, but H-zones and M-lines were occasionally observed in the middle of some sarcomeres (Fig. 4A, B, asterisks). In favorable sections, striated myofibrils were observed inserting into robust, electron-dense adherens junctions at 
the membrane where two cardiomyocytes contact one another closely (Fig. 4A, C, arrows). However, myofibril assembly is asynchronous, and, in some regions, loose bundles of thin and thick filaments were also observed interspersed between skeins of irregularly arranged, electron-dense Z-bodies, likely representing nascent or pre-myofibrils (data not shown) (Markwald, 1973). Nevertheless, we identified myofibrils in every cell examined in the a $T M 1^{+/-}$embryonic hearts (data not shown).

In contrast, in $\mathrm{aTMI}^{-/-}$embryonic hearts, no obvious sarcomeric structures were observed in any of the a $T M 1^{-/-}$cardiomyocytes. Instead, the a $T M 1^{-/-}$cardiomyocytes displayed numerous disordered thick filaments interspersed among a few irregular electron-dense structures resembling Z-bodies with associated thin filaments (Fig. 4D, F, G, white arrowheads). In other regions, large accumulations of scattered thick filaments were observed with no associated Z-bodies (Fig. 4E and data not shown). Also occasionally observed were large, electron-dense, rod-like structures that appeared to have filaments running lengthwise through them and faint periodic banding across their narrow dimension (Fig. 4F, G). Notably, the membranes of adjacent a $T M 1^{-/-}$cardiomyocytes tended not to contact one another over extended distances, and the electron-dense adherens junctions were very small, with few or no attached myofibrils or myofilaments (Fig. 4E, arrows). In some cases, the gaps between cells in regions between the small electron-dense adherens junctions were sizeable, leading to cells with highly elongated processes and extremely irregular shapes (Figs. 2J and 4E and data not shown). Thus, in the absence of a TM1, neither thin nor thick filaments assemble into myofibrils, and adherens junctions are abnormally small, with no attached myofibrils.

\section{aTM1-deficient cardiomyocytes assemble aberrant F-actin fibrils with a-actinin puncta}

To further characterize the filamentous structures containing thick filaments and Z-bodies in the $\mathrm{aTMI}^{-/-}$cardiomyocytes, we performed whole-mount immunofluorescence staining for F-actin and sarcomeric a-actinin in E9.5 hearts. In the a $\mathrm{TMI}^{+/+}$embryonic hearts, cardiomyocytes displayed well-organized myofibrils (Fig. 5A-C, G-I), with typical striated a-actinin at Z-lines (Fig. 4H,I, lines) and striated F-actin with apparent H-zone gaps (Fig. 5G,I, arrowheads), as expected. In contrast, the a $T M 1^{-/}$embryonic hearts failed to form striated myofibrils, and instead assembled F-actin fibrils decorated with puncta of a-actinin along their lengths (Fig. 5DF, J-L). These linear F-actin fibrils displayed irregular variations in F-actin intensity along their lengths, but no distinct H-zone gaps in F-actin (Fig. 5J, L, arrowheads). The increased $\mathrm{F}$-actin intensities coincided with the a-actinin puncta, suggesting that the a-actinin puncta observed in a $\mathrm{TMI}^{-/-}$cardiomyocytes might be immature Z-bodies corresponding to the electron-dense structures observed by TEM (Fig. 4D, F, G). In addition to the linear strings of a-actinin puncta along F-actin fibrils, longer and thicker rods of a-actinin were also observed in some regions, which stained relatively poorly for F-actin (see below). These aberrant F-actin fibrils were most likely due to defective myofibril assembly, rather than degeneration of mature striated myofibrils after assembly, because no striated myofibrils were observed in a $T M 1^{-/-}$hearts even in the earliest stages of morphogenesis (3-5 somites; data not shown). 
One current model of myofibrillogenesis proposes that early pre-myofibrils are characterized by $\mathrm{a}$-actinin puncta forming immature Z-bodies, spaced $\sim 0.75 \mu \mathrm{m}$ apart along continuous F-actin fibrils (Dabiri et al., 1997; Du et al., 2008). Pre-myofibrils then develop into mature myofibrils with a-actinin-containing Z-lines spaced $>1.6 \mu \mathrm{m}$ apart (Dabiri et al., 1997; Sanger et al., 2000; Du et al., 2003; Du et al., 2008). To test whether the filamentous structures in the a $T M 1^{-/-}$embryonic heart might correspond to pre-myofibrils in the normal myofibril assembly pathway, we measured distances between adjacent a-actinin Z-lines (or puncta) in high-magnification images of F-actin- and a-actinin-stained fibrils in E9.5 $\mathrm{aTM1} 1^{+/+}$and $\mathrm{a} T M 1^{-/-}$embryonic hearts (Fig. 5G-L). In aTM1 ${ }^{-/-}$hearts, we measured linear a-actinin/F-actin fibrils with at least 3 collinear a-actinin-staining puncta, and with Factin staining extending beyond $a$-actinin on both ends of the fibril (Fig. 5J-L). While the average $\mathrm{Z}-\mathrm{Z}$ distances were the same between $\mathrm{aTM1^{+/+ }}$ and a TM1 ${ }^{-/-}$hearts $(1.6 \pm 0.09 \mu \mathrm{m}$ $(n=82)$ and $1.6 \pm 0.36 \mu \mathrm{m}(n=45)(p=0.76)$, respectively), the distances between $a$-actinin puncta in the a $T M 1^{-/-}$hearts were much more variable. An F-test for variance between the $\mathrm{aTM1^{+/+ }}$ and $\mathrm{aTM1} 1^{-/-} \mathrm{Z}-\mathrm{Z}$ distances returned $p=2.84 \times 10^{-25}$, indicating that the difference

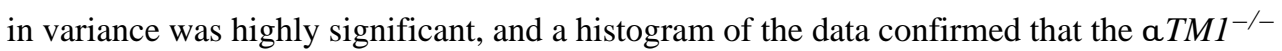
$\mathrm{Z}-\mathrm{Z}$ distances were more widely dispersed than the $\mathrm{a} T M 1^{+/+} \mathrm{Z}-\mathrm{Z}$ distances (Fig. 5M). Thus, while the average $\mathrm{Z}-\mathrm{Z}$ distances are the same in the $\mathrm{a} T M 1^{-/-}$aberrant F-actin fibrils and a TM1 $1^{+/+}$striated myofibrils, the aTM1 ${ }^{-/-} \mathrm{Z}-\mathrm{Z}$ distances are much more variable. Furthermore, the average a $T M 1^{-/-} \mathrm{Z}-\mathrm{Z}$ distance of $\sim 1.6 \mu \mathrm{m}$ is much larger than that measured for pre-myofibrils (Sanger et al., 2000), indicating that the structures in the a $T M 1^{-/-}$embryonic heart are not likely to be pre-myofibrils but, instead, represent aberrant a-actinin/F-actin fibrils.

The giant protein titin is believed to be a key player in the assembly and spacing of Z-bodies (Kontrogianni-Konstantopoulos et al., 2009), and the Z-line region of titin is thought to be one of the earliest assembling components of myofibrils, colocalizing with sarcomeric aactinin in early Z-bodies of nascent and premyofibrils (Peckham et al., 1997; Turnacioglu et al., 1997; Ayoob et al., 2000). To determine whether titin assembly is coordinated with assembly of sarcomeric $\mathrm{a}$-actinin puncta in the aberrant $\mathrm{a}$-actinin/F-actin fibrils in a $T M 1^{-/-}$cardiomyocytes, we immunostained embryonic hearts with antibodies against the Z-line epitope of titin, Z1Z2. In E9.5 a TM1 ${ }^{+/+}$hearts, titin Z1Z2 localized to the Z-lines of striated myofibrils, colocalizing with sarcomeric a-actinin, as expected (Fig. 6A-D, I-M). In contrast, in E9.5 $\mathrm{aTM1}^{-/-}$hearts, titin Z1Z2 staining accumulated in a disorganized fashion in the vicinity of the a-actinin/F-actin fibrils, but failed to consistently colocalize with the a-actinin puncta or rods (Fig. 6E-H). The association of titin Z1Z2 with some but not all of the a-actinin puncta was evident in high-magnification images of regions where individual a-actinin puncta are well resolved (Fig. $6 \mathrm{~N}-\mathrm{W}$ ). We conclude that assembly of a-actinin into Z-bodies is incompletely coordinated with assembly of the Z1Z2 domain of titin, and, therefore, supporting the conclusion that the aberrant $a$-actinin/F-actin fibrils in the aTM1 ${ }^{-/-}$hearts are not premyofibril intermediates along a normal myofibril assembly pathway.

An alternative model to regulate Z-body assembly and spacing is based on assembly of 1.6$\mu \mathrm{m}$-long myosin thick filaments and their interactions with the oppositely polarized actin 
filaments extending from adjacent Z-bodies (Holtzer et al., 1997; Sanger et al., 2005). Our TEM observations of thick filaments in the vicinity of Z-bodies (Fig. 4D-G) raise the possibility that thick filament components may partially assemble with these structures. Therefore, we immunostained embryonic hearts for myomesin, a marker of thick filament assembly that crosslinks myosin thick filaments at the M-line (Agarkova and Perriard, 2005; Schoenauer et al., 2005; Schoenauer et al., 2008). In the myofibrils of $a T M 1^{+/+}$hearts, myomesin localized at the M-line in the middle of the sarcomere, exhibiting a striated pattern of staining alternating with a-actinin at the Z-line, as expected (Fig. 7A-C, G-I). In the $\mathrm{aTMI}^{-/-}$hearts, myomesin accumulated in disorganized clumps in the vicinity of the aactinin/F-actin fibrils (Fig. 7D-F), similar to titin Z1Z2 (Fig. 6E-H), suggesting that thick filament components may assemble, but in a disordered fashion. At higher magnification, in regions where individual a-actinin puncta were well resolved, occasional discontinuous concentrations of myomesin staining were associated with these structures, but, again, failed to exhibit a regular pattern with respect to a-actinin (Fig. 7JL). This was consistent with the TEM of $\mathrm{aTM1}^{-/-}$cardiomyocytes showing scattered thick filaments accumulated around areas with electron-dense Z-bodies (Fig. 4D-G). Therefore, we conclude that 1.6- $\mu \mathrm{m}$-long myosin thick filaments crosslinked by myomesin at the M-line are unlikely to coordinate the assembly and distribution of Z-bodies containing a-actinin along the aberrant F-actin fibrillar structures in the a $\mathrm{TMI}^{-/-}$cardiomyocytes.

\section{aTM1 and Tmod1 exhibit co-dependent assembly into myofibrils}

Tmod1 is an actin-capping protein that binds aTM1 and localizes to the pointed ends of the thin filaments in striated muscle sarcomeres (Gregorio and Fowler, 1995; Gregorio et al., 1995; Littlefield et al., 2001; Fritz-Six et al., 2003; Gokhin and Fowler, 2011). Based on the interdependence of Tmod1-aTM1 effects on F-actin assembly and stability (Weber et al., 1994; Mudry et al., 2003), we reasoned that Tmod1 assembly onto thin filament pointed ends might depend on aTM1 and, conversely, that aTM1 assembly onto thin filaments might depend on Tmod1. In aTM1 ${ }^{+/+}$embryonic hearts, Tmod1 localized to the pointed ends of the thin filaments at the H-zone, alternating with the a-actinin stripes at the Z-line (Fig. 8A-D). By contrast, in aTM1 ${ }^{-/-}$embryonic hearts, Tmod1 exhibited irregular and diffuse staining in the vicinity of the aberrant $a$-actinin/F-actin fibrils and elsewhere in the cytoplasm (Fig. 8E-H). While Tmod1 accumulated near the $\mathrm{a}$-actinin/F-actin fibrils in a $T M 1^{-/-}$embryonic hearts, Tmod1 was not concentrated into puncta, organized in a periodic pattern, or associated with the larger rod-like a-actinin/F-actin structures (Fig. 8E$\mathrm{H})$. This suggests that Tmod1 may not bind tightly to the thin filament pointed ends in the absence of aTM1. This could lead to F-actin instability and filaments with unregulated and variable lengths, consistent with the gradual tapering of F-actin staining intensity further away from some of the a-actinin puncta in the F-actin fibrils (Fig. 5).

Next, to test whether Tmod1 is required for aTM1 assembly into myofibrils, we examined aTM1 localization in $\mathrm{TmodI}^{-/-}$hearts. Tmod1 ${ }^{-/-}$embryos die at E9.5 due to aborted cardiac morphogenesis and lack of beating (Chu et al., 2003; Fritz-Six et al., 2003). Previous studies have shown that $\mathrm{Tmodl}^{-/-}$cardiomyocytes fail to assemble striated myofibrils, with F-actin and sarcomeric a-actinin accumulating in large bundles and rod-like structures in the cytoplasm, and less frequently forming premyofibril-like structures with continuous F-actin 
and closely-spaced a-actinin puncta (Chu et al., 2003; Fritz-Six et al., 2003; McKeown et al., 2008). In $\operatorname{Tmod}^{+/+}$embryonic hearts, a TM1 was localized in a striated pattern with Factin along the myofibrils, as expected (Fig. 8I-L). However, in Tmodl $1^{-/-}$hearts, aTM1 was associated with the regions containing large bundles of F-actin, and also diffuse in the cytoplasm in regions with little or no F-actin (Fig. 8M-P). aTM1 was not detected on the Factin rods that form in the absence of Tmod1 (Fig. 8M-P, red arrowhead). These rods contain sarcomeric a-actinin as well as F-actin (McKeown et al., 2008), and may be similar to the rod-like structures of sarcomeric a-actinin and F-actin observed in aTM1-/embryonic hearts (Fig. 6G, 8G). Collectively, these data show that Tmod1 and aTM1 require one another for normal assembly into myofibrils.

\section{Adherens belts of a-actinin/F-actin fail to assemble in aTM1- or Tmod1-deficient cardiomyocytes}

In addition to myofibril assembly defects, a $T M 1^{-/-}$cardiomyocytes showed defects in cellcell contacts (Fig. 2 $\mathrm{H}^{\prime}, \mathrm{J}$; Fig. 4E). To further investigate this defect, we immunostained E9.5 hearts for the cell junction marker $\mathrm{N}$-cadherin to demarcate cell boundaries (Shiraishi et al., 1993) and imaged the outermost cell layer by confocal microscopy. In a TM1 ${ }^{+/+}$ embryos, $\mathrm{N}$-cadherin outlined the cardiomyocytes in the outer wall of the heart, revealing cell boundaries arranged in a continuous cobblestone pattern, similar to an epithelial sheet (Fig. 9A) (Shiraishi et al., 1993). The junctions of the a $T M 1^{-/-}$cardiomyocytes also stained brightly for $\mathrm{N}$-cadherin, indicating that their cadherin-based adhesive junctions were present. Both membrane and cytoplasmic staining of $\mathrm{N}$-cadherin were brighter in the a TM1 ${ }^{-/-}$cardiomyocytes, suggesting compensatory upregulation of N-cadherin to stabilize adhesive junctions (Fig. 9B). However, a $T M 1^{-/}$cardiomyocytes displayed highly irregular shapes with convoluted contours (Fig. 9B, arrowheads), suggesting defects in cell adhesion and morphology in the absence of aTM1, consistent with the TEM images (Figs. 2J, 4E). Moreover, in the a TM1 ${ }^{-/-}$hearts, the $\mathrm{N}$-cadherin staining imaged in a single optical section revealed apparent gaps in the tissue (Fig. 9B, asterisks), which likely corresponded to the wrinkled surface of the myocardium observed in the gross morphology of the embryos (Figs. 2 and 3B, and data not shown). This is unlike $\mathrm{a} T M 1^{+/+}$hearts, where the $\mathrm{N}$-cadherin junctions and the cardiomyocytes were all in the same plane on the outer surface (Fig. 9A), corresponding to a relatively smooth myocardial surface (Fig. 2) (Shiraishi et al., 1993). To quantify the tortuous contours of the $\mathrm{N}$-cadherin staining on the a $T M 1^{-/-}$cardiomyocyte membranes, we measured the linearity indices of several cells in both $a \mathrm{TM}^{+/+}$and aTM1 ${ }^{-/-}$embryos. In this assay, the contour length of the cell membrane between two vertices (the intersection points formed at the contacts between 3 cells) was divided by the shortest distance between the vertices (Otani et al., 2006). If the cell boundaries were relatively straight, then the linearity index was close to 1 , as seen in $\mathrm{a} T M 1^{+/+}$hearts (Fig. 9C). Conversely, if the cell boundaries were tortuous, then the linearity index was greater than 1, as observed in aTM1 ${ }^{-/-}$hearts (Fig. 9C). Thus, while aTM1 is dispensable for Ncadherin assembly at the membrane, aTM1 is required for normal cell boundaries and embryonic cardiomyocyte shapes.

$\mathrm{N}$-cadherin adherens junctions at the apical poles of cardiomyocytes in the embryonic heart are stabilized by circumferential belts of sarcomeric a-actinin and F-actin that form a 
continuous nonstriated ring around the periphery of each cardiomyocyte (Schultheiss et al., 1990; Shiraishi et al., 1993). The continuous adherens belts of sarcomeric a-actinin and Factin can be visualized in the outer $2 \mu \mathrm{m}$ of the cells at the surface of the myocardium, and are distinct from the striated myofibrils that traverse the cell interior (Fig. 10A-C). Strikingly, in the a $\mathrm{TM1}^{-/-}$embryonic hearts, the continuous belts of sarcomeric a-actinin and F-actin demarcating the cell boundaries were absent (Fig. 10D-F). Instead, the aTM1-/cardiomyocytes displayed numerous and irregularly arranged F-actin fibrils with puncta of sarcomeric a-actinin (Fig. 10D-F), similar to the abnormal structures in the cardiomyocyte interior (Fig. 5). In addition, some F-actin- and a-actinin-containing rod-like structures were occasionally observed near the cell surface in the a $T M 1^{-1-}$ embryonic cardiomyocytes (Fig. 10D-F, arrowheads). Thus, while N-cadherin assembles at the membrane (Fig. 9B) and small adherens junctions form in the absence of aTM1 (Fig. 4E), aTM1 is required for assembly of sarcomeric a-actinin and F-actin into continuous circumferential adherens belts at the cell periphery near the myocardial surface.

The interdependence of aTM1 and Tmod1 assembly into myofibrils (Fig. 8) suggests that absence of Tmod1, which leads to impaired aTM1 assembly (Fig. 8M-P), might also result in defective adherens belts, similar to the absence of aTM1 (Fig. 10D-F). Indeed, examination of the outer myocardial surfaces of $\mathrm{Tmodl}^{-/-}$embryonic hearts revealed a complete lack of continuous adherens belts of sarcomeric a-actinin and F-actin (Fig. 10G-I). Instead, the sarcomeric a-actinin assembled into numerous thick rod-like structures that stained weakly for F-actin (Fig. 10G-I, arrowheads), and F-actin additionally assembled into large aggregates that stained poorly for a-actinin (Fig. 10G-I, asterisks). Thus, absence of either aTM1 or Tmod1 prevents assembly of continuous adherens belts of a-actinin and Factin, further highlighting the overlapping functions of aTM1 or Tmod1 in cardiomyocytes. However, the phenotypes are distinct in that the large $a$-actinin/F-actin rods are considerably more abundant in the $\mathrm{Tmodl}^{-/-}$hearts than in a $T M \mathrm{I}^{-/-}$hearts. Likewise, a $T M 1^{-1-}$ hearts contain abundant long, thin F-actin fibrils with a-actinin puncta, which are less frequently observed in Tmodl ${ }^{-/-}$hearts (compare Fig. 10D with 10G; Fig. 5; also see (Fritz-Six et al., 2003; McKeown et al., 2008)).

\section{Discussion}

The study is the first to illuminate roles for aTM1 in mammalian myofibril assembly and cardiac development by a comprehensive analysis of the phenotype of hearts from aTM1deficient mice, using a combination of histology, TEM imaging, and immunofluorescence localization of myofibril assembly markers. Here, we demonstrate that mice lacking a TM1 are embryonic lethal at E9.5, due to profound defects in cardiac development, loss of beating, failure of myofibril assembly, and lack of normal thick and thin filament interactions. Our work extends previous studies on TM-deficient axolotl and zebrafish hearts by showing that cardiomyocytes from a TM1-deficient mouse hearts also fail to assemble striated myofibrils. A novel finding is that aTM1-deficient cardiomyocytes display aberrant non-striated F-actin fibrils with highly variable Z-body spacing, indicating that aTM1 is required for regular sarcomere periodicity. In addition, comparison of aTM- and Tmod1deficient cardiomyocytes revealed that Tmod1 and aTM1 depend on one another for assembly into thin filaments. Moreover, unexpectedly, both myomesin-containing thick 
filaments as well as titin Z1Z2 fail to assemble in a striated pattern, highlighting interdependence of thick and thin filament assembly.

Our work is also the first to define a new function for aTM1 in cell shape and cell adhesion by showing that a TM1-deficient hearts have defects, with lack of formation of adherens belts at apical poles of cardiomyocytes. Importantly, deficiency of either aTM1 or its binding partner Tmod1 impairs F-actin stability in adherens belts, pointing to a novel aTM1/Tmod1-based pathway in regulating the stability of F-actin in the heart. These data point to key requirements for a TM1 in myofibrillogenesis, adhesion, and morphogenesis in the developing heart, and suggest novel molecular mechanisms of aTM1-based cardiomyopathies. An important limitation of our study is that we cannot readily distinguish whether the observed myofibril and adherens belt disorganization phenotypes are primary effects of aTM1 deletion, or secondary effects due to global loss of cardiac function. Future studies will benefit from examination of myofibril assembly and adherens junctions at earlier developmental stages, as well as in experimental models of congenital cardiomyopathies arising from TPMI gene defects.

\section{aTM1 function in myofibril assembly and cardiac development}

Comparison of the a $\mathrm{TMI}^{-/-}$phenotype reported here with the phenotype of cardiac mutant axolotls is instructive for elucidating the mechanisms of a TM1 function in cardiac myofibril assembly. In the cardiac mutant axolotl heart, absence of aTM1 leads to widespread abnormalities in the targeting and organization of thin filament and sarcomere-associated proteins during cardiac development, leading to failure of myofibril assembly and a lack of contraction and beating (Lemanski, 1973; Lemanski, 1979; Lemanski et al., 1980; Fuldner et al., 1984; Starr et al., 1989; Erginel-Unaltuna and Lemanski, 1994; La France and Lemanski, 1994; Zajdel et al., 1998; Zajdel et al., 1999; McLean et al., 2006; Zajdel et al., 2007). This phenotype is remarkably similar to that observed in the a $T M 1^{-/-}$mouse heart described here. Similarly, deletion of cardiac troponin $\mathrm{T}$ in mice results in embryonic lethality at $\hat{E} 10$ due to impaired cardiac myofibril assembly, sarcomere disruption, an abnormally thin cardiac wall with reduced trabeculae, and lack of heartbeat (Nishii et al., 2008), quite similar to the $\mathrm{aTMI}^{-/-}$mouse heart. Troponin $\mathrm{T}$ is also essential for myofibril assembly in zebrafish striated muscles (Sehnert et al., 2002; Huang et al., 2009; Ferrante et al., 2011). It is noteworthy that the defects in myofibril assembly reported for the zebrafish troponin $\mathrm{T}$ mutants are accompanied by reduced assembly of aTM1 onto thin filaments (Sehnert et al., 2002; Ferrante et al., 2011), indicating that troponins are required for stable aTM1 association with thin filaments. Thus, secondary loss of a TM1 may partly account for the myofibril assembly defects observed in the troponin $\mathrm{T}$ mutants. Indeed, in vitro biochemical studies have shown that troponin T strengthens a TM1 binding to F-actin, thereby enhancing aTM1's ability to stabilize F-actin from depolymerization and severing (Wegner and Walsh, 1981; Broschat, 1990; Weigt et al., 1990; Hill et al., 1992). On the other hand, deletion of cardiac troponin I in mice does not result in defects in embryonic cardiac myofibril assembly, likely due to compensation by fetal troponin I (Huang et al., 1999).

A number of previous studies have suggested that thin and thick filament assembly are independent (Markwald, 1973; Epstein and Fischman, 1991; Epstein and Bernstein, 1992; 
Rudy et al., 2001; Fritz-Six et al., 2003; Kontrogianni-Konstantopoulos et al., 2006), but our data suggest that this is not the case for myofibrils assembling in the embryonic mouse heart. In the absence of aTM1, sarcomeric F-actin assembles into long, linear fibrils with aactinin puncta along their lengths, but, while myomesin accumulates in the vicinity of these fibrils, it is not organized into an alternating pattern with the a-actinin puncta. TEM also indicates that thick filaments are interspersed with Z-bodies in aTM1-deficient cardiomyocytes, but their organization and localization are irregular. Other studies have proposed that the thick filaments are the earliest myofibril components to become striated independent of thin filaments (Wang et al., 1988; Guan et al., 1999), yet our data argue against that, as no striated myomesin or thick filaments were observed in aTM1-deficient hearts. Therefore, aTM1 is required to achieve assembly of organized thick filaments during myofibrillogenesis. Titin has been proposed act as a thick filament scaffold during this process (Kontrogianni-Konstantopoulos et al., 2009), and, therefore, one possibility is that failure of proper titin incorporation (as shown by nonstriated immunostaining for titin Z1Z2) causes lack of thick filament organization.

An alternative explanation for impaired thick filament organization is that, in absence of aTM1, most of the F-actin colocalizes with the a-actinin puncta, consistent with $a$-actinin binding along more of the actin filaments, resulting in insufficient thin filament extensions with accessible myosin binding sites. This agrees with the presence of many expanded aactinin rod-like structures rather than narrow Z-lines in aTM1-deficient cardiomyocytes. This phenotype is also expected from in vitro studies showing that aTM1 inhibits a-actinin binding to F-actin (Goll et al., 1972; Stromer and Goll, 1972). Thus, insufficient association of myosin thick filaments with F-actin in the absence of aTM1 may impair the transition of the punctate $a$-actinin/F-actin fibrils into striated myofibrils. Moreover, unregulated actomyosin contractility may cause degeneration of partially assembled myofibrils in aTM1-deficient hearts, as observed in troponin I and T mutants (Huang et al., 1999; Sehnert et al., 2002; Nongthomba et al., 2004; Nishii et al., 2008; Ferrante et al., 2011). These conclusions agree with studies in cultured myocytes suggesting that actomyosin contractility is important for formation and maintenance of myofibrils (Soeno et al., 1999; De Deyne, 2000; Ramachandran et al., 2003; Kagawa et al., 2006).

\section{Co-dependent assembly of aTM1 and Tmod1 in cardiac myofibrils}

The pointed-end capping protein Tmod 1 binds to a TM1 and enhances the ability of aTM1 to stabilize F-actin (Weber et al., 1994; Weber et al., 1999). In a complementary fashion, the presence of a TM1 on F-actin enhances Tmod1's capping and stabilization of F-actin pointed ends (Weber et al., 1994). Moreover, antibody inhibition studies in cultured cardiomyocytes have demonstrated that loss of the aTM1-Tmod1 interaction results in a dramatic disassembly of the thin filaments and subsequent loss of contractile activity (Mudry et al., 2003). Our data indicate that aTM1 and Tmod1 also cooperate to promote Factin stability in vivo, since Tmod1 fails to assemble along the F-actin fibrils in aTM1deficient hearts - an observation consistent with failure of Tmod1 assembly in cardiac mutant axolotl hearts (McLean et al., 2006) - and, likewise, a TM1 fails to assemble along F-actin fibrils in Tmod1-deficient hearts. Mutually cooperative binding of Tmod1 and aTM1 to F-actin may promote polymerization of stable, length-regulated thin filaments 
extending from a-actinin-containing Z-bodies during myofibril assembly. Interdependence of aTM1 and Tmod1 in stabilizing F-actin structures is highlighted by the failure of continuous, membrane-associated adherens belts of sarcomeric a-actinin and F-actin to form in the absence of either aTM1 or Tmod1 in the embryonic heart.

Despite common functions of aTM1 and Tmod1 in stabilizing F-actin, loss of Tmod1 has a different developmental phenotype in the embryonic heart than does loss of aTM1. Heart development in the absence of Tmod1 is arrested at the stage of looping morphogenesis (E8.5), and the Tmod1-deficient heart tube twitches asynchronously rather than beats regularly (Chu et al., 2003; Fritz-Six et al., 2003; McKeown et al., 2008). By contrast, the aTM1-deficient heart loops and appears to form chambers; however, these chambers expand abnormally up to E10.5, leading to large dysmorphic hearts that fail to twitch or beat. These differences in phenotypes may arise from unique aTM1 or Tmod 1 functions in myofibril assembly or crosstalk with other developmental processes. For example, Tmod1deficient cardiomyocytes assemble F-actin fibrils and occasional myomesin striations, suggesting that some myofibrils achieve contractile function, explaining the twitching of the Tmod1-deficient heart tubes (Fritz-Six et al., 2003). However, the aberrant a actinin F-actin fibrils in aTM1-deficient hearts do not contain myomesin striations or organized thick filaments. Hence, aTM1 and Tmod1 appear to share common functions in F-actin stabilization during myofibril assembly, but distinct functions in regulating productive thin and thick filament interactions, with aTM1 but not Tmod1 required for the latter. In addition, the inability of Tmod1-deficient hearts to progress past the looping stage suggests that Tmod1 has unique functions at an earlier developmental stage than aTM1.

The actin cytoskeleton plays key roles in the morphogenesis of the early heart tube (looping morphogenesis) and in myofibril assembly and contraction in cardiomyocytes. It has been proposed that looping morphogenesis, the process by which the linear heart tube becomes looped, resulting in chamber specification, is dependent on myofibril assembly and organization in the cardiomyocytes of the early heart tube (Manasek, 1981). Studies have shown that while actin polymerization is required for cardiac looping morphogenesis (Itasaki et al., 1989; Shiraishi et al., 1992; Latacha et al., 2005), actomyosin-based contractile function is not (Remond et al., 2006). These results, in conjunction with our findings that both Tmod1- and aTM1-deficient mouse hearts exhibit aberrant myofibril assembly with defects in looping and chamber morphology, highlight the importance of myofibril assembly in cardiac morphogenesis.

\section{aTM1 function in cell-cell adhesion, shape maintenance, and heart size}

A surprising finding of this study is that aTM1 is required for cardiomyocyte shape maintenance and regulation of heart size. aTM1-deficient embryos have enlarged heart tubes with abnormal constrictions and wrinkled surfaces, which are not due to increased cell number in the heart. Instead, the enlarged hearts are due to defects in cell shape and cell adhesion, with reduced adherens junctions and highly expanded spaces between the cardiomyocytes, evidenced by both TEM and histology. To the best of our knowledge, this is a novel phenotype in the mouse heart. One possible explanation for this phenotype is that aTM1 may control cell shape and adhesion indirectly via its function in myofibril assembly. 
Association of striated myofibrils with adherens junctions plays a role in maintenance of cardiomyocyte shape during embryonic heart development (Auman et al., 2007) as well as in the adult heart (Ferreira-Cornwell et al., 2002; Kostetskii et al., 2005). Therefore, loss of aTM1 with concomitant failure of myofibril assembly could, in turn, be responsible for attenuated assembly of adherens junctions, leading to the observed morphological defects. Alternatively, aTM1 may directly control cell shape and adhesion by stabilizing the circumferential adherens belts of sarcomeric a-actinin and F-actin located at the apical poles of the cardiomyocytes. TM isoforms localize to cell-cell junctions in epithelia (Temm-Grove et al., 1998; Dalby-Payne et al., 2003; Bakin et al., 2004; Weber et al., 2007; Nowak et al., 2009), and other TMs have been implicated in human epithelial cell cancers (Stehn et al., 2006; Helfman et al., 2008), suggesting critical roles for TMs in the stability and function of cell-cell junctions. Lack of adherens belts providing circumferential tension may explain the convoluted contours of the cardiomyocyte membranes evidenced by $\mathrm{N}$-cadherin staining in aTM1-deficient hearts, as well as the large spaces between neighboring cells.

It is most likely that the absence of contractile force underlies how deletion of aTM1 leads to defective adherens junctions and abnormal cell shapes. Indeed, mechanical forces have been shown to drive assembly of focal contacts, adhesive structures, and myofibrils themselves in cardiomyocytes (Sharp et al., 1993; Sharp et al., 1997; Yu and Russell, 2005). Because aTM1-deficient cardiomyocytes exhibit a failure of actomyosin-based contractility, it is conceivable that this lack of force generation blocks adherens junction formation and induces abnormal cell shape alterations. In zebrafish, actomyosin-based contractility in cardiomyocytes restricts such cell shape alterations, likely by imparting mechanical robustness or stiffness to the cell (Auman et al., 2007). Analogously, actomyosin-based contractility has also been proposed to control the biconcave shape of mature erythrocytes (Fowler, 1986) and the polarized architecture and morphogenesis of epithelial sheets (Lecuit and Lenne, 2007; Gorfinkiel and Blanchard, 2011).

\section{Experimental Procedures}

\section{Mice and genotyping}

The targeted aTM1 deletion in the TPM1 gene was generated previously (Rethinasamy et al., 1998) and maintained as heterozygotes. aTM1 ${ }^{+/-}$mice were backcrossed onto the C57B1/6J background for at least 6 generations, to 97\% congenicity. Mouse genotypes were identified by PCR using the following primer sets: TmKO ${ }^{\text {fwd }}$

CTCATCTGTTACACTGAAACTCTTGG and TmKOrev

CATGTTTCAGCAGTGTTGGCTG, which correspond to sequences in exon 9a of the TPM1 gene and the inserted HPRT gene, respectively, and result in a 650-bp band in the targeted allele; TmWT ${ }^{\text {fwd }}$ CATCTCTGCCTTCCACTTCCTG and TmWTrev

CCTGCTCTGGAATTCATAACAAGG, which give a 900-bp band from the wild-type allele corresponding to the targeted/deleted region of exon $9 \mathrm{a}$. Timed matings of aTM1 mice were performed to obtain a $T M 1^{+/+}, \mathrm{aTM1}^{+/-}$, and $\mathrm{aTM1^{-/- }}$ mice. The targeted Tmodl gene deletion was also generated previously (Fritz-Six et al., 2003; McKeown et al., 2008), and timed matings of Tmod1 ${ }^{+/-}$mice were performed to obtain TmodI $^{+/+}$and TmodI $^{-/-}$mice. All experiments were performed according to NIH animal care guidelines, 
as approved and enforced by the Institutional Animal Care and Use Committee at The Scripps Research Institute.

\section{Western blotting}

Embryonic tails were removed for genotyping, and the remaining portion of the embryo was snap-frozen in liquid nitrogen and stored at $-80^{\circ} \mathrm{C}$ until the embryos were genotyped (up to 1 week). Then $30 \mu \mathrm{l}$ of $2 \times$ Laemmli sample buffer was added, vortexed for $10 \mathrm{sec}$, sonicated for $30 \mathrm{sec}$, boiled for $10 \mathrm{~min}$, and the sample was split and loaded into 2 lanes on different halves of the same gel. Proteins were separated on 12.5\% SDS-PAGE gels and transferred to nitrocellulose. Antibodies were a mouse monoclonal to exon 9a of aTM1 (CH1;

Developmental Studies Hybridoma Bank, University of Iowa, Iowa City, IA), and a panactin mouse monoclonal (C4; kindly provided by J.L. Lessard, Cincinnati Children's Hospital Medical Center, Cincinnati, OH). HRP-goat anti-mouse IgG and HRP-goat antirabbit IgG were used for secondary detection, and bands were visualized by enhanced chemiluminescence.

\section{Embryonic morphology and histology}

Timed pregnant mice were sacrificed, and embryos were dissected from the uterus in PBS $(\mathrm{pH} 7.4)+0.1 \%$ Triton X-100. For morphological analysis, embryos were staged by somite pair number and imaged live on an Olympus SZ-PT dissecting microscope with a Nikon CoolPix 990 camera. For histology, embryos were fixed in 10\% formalin overnight, washed in $70 \%$ ethanol, dehydrated in an ethanol series, and then mounted in $3 \%$ agarose prior to processing for paraffin embedding, sectioning, and staining with hematoxylin and eosin. Multiple serial sections were stained and analyzed for each time point and genotype.

\section{Immunofluorescence}

Whole-mount immunofluorescence was performed as previously described (McKeown et al., 2008). Antisera and fluorophores were as follows: mouse monoclonal anti-a-actinin (EA53; 1:200; Sigma-Aldrich), mouse monoclonal antibody to exon 1b of aTM1 (TM311; 1:200; Sigma-Aldrich), affinity-purified rabbit polyclonal anti-human Tmod1 (R1749; 10 $\mathrm{ug} / \mathrm{ml}$ ), rabbit polyclonal anti-myomesin (EH; 1:1000; kindly provided by E. Ehler, Kings College London, UK), affinity-purified rabbit polyclonal anti-titin (Z1Z2; 1:100; kindly provided by C.C. Gregorio, University of Arizona, Tucson, AZ), rat monoclonal anti-Ncadherin hybridoma supernatant (1:10; kindly provided by D. Vestweber, Max-Planck Institute, Münster, Germany), rabbit anti-phospho-histone H3 serum (1:500, kindly provided by K.F. Sullivan, National University of Ireland, Galway, Ireland), rhodamine-phalloidin (1:500; Sigma-Aldrich), Alexa-647-phalloidin (1:200; Invitrogen), and Hoechst dye (1:1000; Sigma-Aldrich). Fluorescent secondary antibodies were: Alexa 488-goat anti-rabbit IgG (1:200; Invitrogen), Alexa 546-goat anti-mouse IgG (1:200, Invitrogen), Alexa 647-goat anti-mouse IgG (1:200; Invitrogen), and Cy2-donkey anti-rat IgG (1:200; kindly provided by C.M. Waterman-Storer, National Institutes of Health, Bethesda, MD). 


\section{Confocal microscopy and image analysis}

Z-series and single optical sections were collected on a Bio-Rad 2100 Radiance laserscanning confocal microscope mounted on a Nikon microscope using a 100× (NA 1.3) objective, or on a Zeiss LSM 710 confocal microscope using a 100× (NA 1.4) objective. For $\mathrm{Z}$-series, images were over-sampled such that images were captured using $0.2-\mu \mathrm{m}$ steps with an optical section thickness of $0.8 \mu \mathrm{m}$. Volume reconstructions and measurements were performed using Volocity 6.2 (Improvision, Perkin-Elmer). When necessary, fluorescent images from different color channels were manually aligned based on images of Z-stacks of fluorescent beads. Images were compiled using Adobe Photoshop, and figures were constructed in Adobe Illustrator.

\section{TEM}

Embryos were dissected in PBS (pH 7.2), temporarily placed in 3\% PFA in $0.2 \mathrm{M}$ Hepes ( $\mathrm{pH}$ 7.2), and then fixed in $4 \%$ PFA $+1.5 \%$ glutaraldehyde in $0.1 \mathrm{M}$ cacodylate buffer $(\mathrm{pH}$ 7.4) on ice. Embryos were then processed and imaged on a Philips CM100 transmission electron microscope equipped with an Olympus Megaview III CCD camera as previously described (Gokhin et al., 2010).

\section{Statistics}

Differences between means were detected using Student's $t$-test, and differences between variances were detected using the F-test. Statistical significance was defined as $p<0.05$. Statistical analysis was performed in Microsoft Excel.

\section{Acknowledgments}

We thank Malcolm R. Wood for assistance with electron microscopy and Margie Chadwell for assistance with histology.

Grant sponsorship: This work was supported by NIH/NHLBI grant R01-HL083464 (to V.M.F.), NIH/NIAMS grant P30-AR061303 (to V.M.F.), NIH/NHLBI training grant T32-HL07695 (to C.R.M.), a postdoctoral fellowship from George E. Hewitt Foundation for Medical Research (to C.R.M.), a development grant from the Muscular Dystrophy Association (to D.S.G.), and NIH/NEI core grant P30-EY12598 for image processing and analysis.

\section{References}

Agarkova I, Perriard JC. The M-band: an elastic web that crosslinks thick filaments in the center of the sarcomere. Trends Cell Biol. 2005; 15:477-485. [PubMed: 16061384]

Auman HJ, Coleman H, Riley HE, Olale F, Tsai HJ, Yelon D. Functional modulation of cardiac form through regionally confined cell shape changes. PLoS Biol. 2007; 5:e53. [PubMed: 17311471]

Auman HJ, Yelon D. Vertebrate organogenesis: getting the heart into shape. Curr Biol. 2004; 14:R152-153. [PubMed: 15027466]

Ayoob JC, Turnacioglu KK, Mittal B, Sanger JM, Sanger JW. Targeting of cardiac muscle titin fragments to the Z-bands and dense bodies of living muscle and non-muscle cells. Cell Motil Cytoskeleton. 2000; 45:67-82. [PubMed: 10618168]

Bakin AV, Safina A, Rinehart C, Daroqui C, Darbary H, Helfman DM. A critical role of tropomyosins in TGF-beta regulation of the actin cytoskeleton and cell motility in epithelial cells. Mol Biol Cell. 2004; 15:4682-4694. [PubMed: 15317845]

Bernstein BW, Bamburg JR. Tropomyosin binding to F-actin protects the F-actin from disassembly by brain actin-depolymerizing factor (ADF). Cell Motil. 1982; 2:1-8. [PubMed: 6890875] 
Blanchard EM, Iizuka K, Christe M, Conner DA, Geisterfer-Lowrance A, Schoen FJ, Maughan DW, Seidman CE, Seidman JG. Targeted ablation of the murine alpha-tropomyosin gene. Circ Res. 1997; 81:1005-1010. [PubMed: 9400381]

Blanchoin L, Pollard TD, Hitchcock-DeGregori SE. Inhibition of the Arp2/3 complex-nucleated actin polymerization and branch formation by tropomyosin. Curr Biol. 2001; 11:1300-1304. [PubMed: 11525747]

Broschat KO. Tropomyosin prevents depolymerization of actin filaments from the pointed end. J Biol Chem. 1990; 265:21323-21329. [PubMed: 2250026]

Broschat KO, Weber A, Burgess DR. Tropomyosin stabilizes the pointed end of actin filaments by slowing depolymerization. Biochemistry. 1989; 28:8501-8506. [PubMed: 2605200]

Burgess DR, Broschat KO, Hayden JM. Tropomyosin distinguishes between the two actin-binding sites of villin and affects actin-binding properties of other brush border proteins. J Cell Biol. 1987; 104:29-40. [PubMed: 3793760]

Chacko KJ. Observations on the ultrastructure of developing myocardium of rat embryos. J Morphol. 1976; 150:681-709. [PubMed: 1003489]

Chu X, Chen J, Reedy MC, Vera C, Sung KL, Sung LA. E-Tmod capping of actin filaments at the slow-growing end is required to establish mouse embryonic circulation. Am J Physiol Heart Circ Physiol. 2003; 284:H1827-1838. [PubMed: 12543641]

Clark KA, McElhinny AS, Beckerle MC, Gregorio CC. Striated muscle cytoarchitecture: an intricate web of form and function. Annu Rev Cell Dev Biol. 2002; 18:637-706. [PubMed: 12142273]

Dabiri GA, Turnacioglu KK, Sanger JM, Sanger JW. Myofibrillogenesis visualized in living embryonic cardiomyocytes. Proc Natl Acad Sci U S A. 1997; 94:9493-9498. [PubMed: 9256510]

Dalby-Payne JR, O'Loughlin EV, Gunning P. Polarization of specific tropomyosin isoforms in gastrointestinal epithelial cells and their impact on CFTR at the apical surface. Mol Biol Cell. 2003; 14:4365-4375. [PubMed: 12960432]

De Deyne PG. Formation of sarcomeres in developing myotubes: role of mechanical stretch and contractile activation. Am J Physiol Cell Physiol. 2000; 279:C1801-1811. [PubMed: 11078695]

Du A, Sanger JM, Linask KK, Sanger JW. Myofibrillogenesis in the first cardiomyocytes formed from isolated quail precardiac mesoderm. Dev Biol. 2003; 257:382-394. [PubMed: 12729566]

Du A, Sanger JM, Sanger JW. Cardiac myofibrillogenesis inside intact embryonic hearts. Dev Biol. 2008; 318:236-246. [PubMed: 18455713]

Epstein HF, Bernstein SI. Genetic approaches to understanding muscle development. Dev Biol. 1992; 154:231-244. [PubMed: 1426637]

Epstein HF, Fischman DA. Molecular analysis of protein assembly in muscle development. Science. 1991; 251:1039-1044. [PubMed: 1998120]

Erginel-Unaltuna N, Lemanski LF. Immunofluorescent studies on titin and myosin in developing hearts of normal and cardiac mutant axolotls. J Morphol. 1994; 222:19-32. [PubMed: 7966344]

Ferrante MI, Kiff RM, Goulding DA, Stemple DL. Troponin T is essential for sarcomere assembly in zebrafish skeletal muscle. J Cell Sci. 2011; 124:565-577. [PubMed: 21245197]

Ferreira-Cornwell MC, Luo Y, Narula N, Lenox JM, Lieberman M, Radice GL. Remodeling the intercalated disc leads to cardiomyopathy in mice misexpressing cadherins in the heart. J Cell Sci. 2002; 115:1623-1634. [PubMed: 11950881]

Fowler VM. An actomyosin contractile mechanism for erythrocyte shape transformations. J Cell Biochem. 1986; 31:1-9. [PubMed: 3722275]

Fritz-Six KL, Cox PR, Fischer RS, Xu B, Gregorio CC, Zoghbi HY, Fowler VM. Aberrant myofibril assembly in tropomodulin 1 null mice leads to aborted heart development and embryonic lethality. J Cell Biol. 2003; 163:1033-1044. [PubMed: 14657235]

Fuldner RA, Lim SS, Greaser ML, Lemanski LF. Accumulation and localization of troponin-T in developing hearts of Ambystoma mexicanum. J Embryol Exp Morphol. 1984; 84:1-17. [PubMed: 6442732]

Gokhin DS, Fowler VM. Tropomodulin capping of actin filaments in striated muscle development and physiology. J Biomed Biotechnol. 2011; 2011:103069. [PubMed: 22013379] 
Gokhin DS, Lewis RA, McKeown CR, Nowak RB, Kim NE, Littlefield RS, Lieber RL, Fowler VM. Tropomodulin isoforms regulate thin filament pointed-end capping and skeletal muscle physiology. J Cell Biol. 2010; 189:95-109. [PubMed: 20368620]

Goll DE, Suzuki A, Temple J, Holmes GR. Studies on purified alpha-actinin. I. Effect of temperature and tropomyosin on the alpha-actinin/F-actin interaction. J Mol Biol. 1972; 67:469-488. [PubMed: 5045308]

Gorfinkiel N, Blanchard GB. Dynamics of actomyosin contractile activity during epithelial morphogenesis. Curr Opin Cell Biol. 2011; 23:531-539. [PubMed: 21764278]

Gregorio CC, Antin PB. To the heart of myofibril assembly. Trends Cell Biol. 2000; 10:355-362. [PubMed: 10932092]

Gregorio CC, Fowler VM. Mechanisms of thin filament assembly in embryonic chick cardiac myocytes: tropomodulin requires tropomyosin for assembly. J Cell Biol. 1995; 129:683-695. [PubMed: 7730404]

Gregorio CC, Weber A, Bondad M, Pennise CR, Fowler VM. Requirement of pointed-end capping by tropomodulin to maintain actin filament length in embryonic chick cardiac myocytes. Nature. 1995; 377:83-86. [PubMed: 7544875]

Guan K, Furst DO, Wobus AM. Modulation of sarcomere organization during embryonic stem cellderived cardiomyocyte differentiation. Eur J Cell Biol. 1999; 78:813-823. [PubMed: 10604658]

Gunning P, O’Neill G, Hardeman E. Tropomyosin-based regulation of the actin cytoskeleton in time and space. Physiol Rev. 2008; 88:1-35. [PubMed: 18195081]

Helfman DM, Flynn P, Khan P, Saeed A. Tropomyosin as a regulator of cancer cell transformation. Adv Exp Med Biol. 2008; 644:124-131. [PubMed: 19209818]

Hill LE, Mehegan JP, Butters CA, Tobacman LS. Analysis of troponin-tropomyosin binding to actin. Troponin does not promote interactions between tropomyosin molecules. J Biol Chem. 1992; 267:16106-16113. [PubMed: 1644797]

Holtzer H, Hijikata T, Lin ZX, Zhang ZQ, Holtzer S, Protasi F, Franzini-Armstrong C, Sweeney HL. Independent assembly of 1.6 microns long bipolar MHC filaments and I-Z-I bodies. Cell Struct Funct. 1997; 22:83-93. [PubMed: 9113394]

Huang W, Zhang R, Xu X. Myofibrillogenesis in the developing zebrafish heart: A functional study of tnnt2. Dev Biol. 2009; 331:237-249. [PubMed: 19427304]

Huang X, Pi Y, Lee KJ, Henkel AS, Gregg RG, Powers PA, Walker JW. Cardiac troponin I gene knockout: a mouse model of myocardial troponin I deficiency. Circ Res. 1999; 84:1-8. [PubMed: 9915769]

Ishikawa R, Yamashiro S, Matsumura F. Differential modulation of actin-severing activity of gelsolin by multiple isoforms of cultured rat cell tropomyosin. Potentiation of protective ability of tropomyosins by 83-kDa nonmuscle caldesmon. J Biol Chem. 1989; 264:7490-7497. [PubMed: 2540194]

Itasaki N, Nakamura H, Yasuda M. Changes in the arrangement of actin bundles during heart looping in the chick embryo. Anat Embryol (Berl). 1989; 180:413-420. [PubMed: 2619084]

Kagawa M, Sato N, Obinata T. Effects of BTS (N-benzyl-p-toluene sulphonamide), an inhibitor for myosin-actin interaction, on myofibrillogenesis in skeletal muscle cells in culture. Zoolog Sci. 2006; 23:969-975. [PubMed: 17189909]

Kobayashi T, Solaro RJ. Calcium, thin filaments, and the integrative biology of cardiac contractility. Annu Rev Physiol. 2005; 67:39-67. [PubMed: 15709952]

Kontrogianni-Konstantopoulos A, Ackermann MA, Bowman AL, Yap SV, Bloch RJ. Muscle giants: molecular scaffolds in sarcomerogenesis. Physiol Rev. 2009; 89:1217-1267. [PubMed: 19789381]

Kontrogianni-Konstantopoulos A, Catino DH, Strong JC, Bloch RJ. De novo myofibrillogenesis in C2C12 cells: evidence for the independent assembly of M bands and Z disks. Am J Physiol Cell Physiol. 2006; 290:C626-637. [PubMed: 16207790]

Kostetskii I, Li J, Xiong Y, Zhou R, Ferrari VA, Patel VV, Molkentin JD, Radice GL. Induced deletion of the $\mathrm{N}$-cadherin gene in the heart leads to dissolution of the intercalated disc structure. Circ Res. 2005; 96:346-354. [PubMed: 15662031]

Kostyukova AS, Hitchcock-DeGregori SE. Effect of the structure of the N terminus of tropomyosin on tropomodulin function. J Biol Chem. 2004; 279:5066-5071. [PubMed: 14660556] 
La France S, Lemanski LF. Immunofluorescent confocal analysis of tropomyosin in developing hearts of normal and cardiac mutant axolotls, Ambystoma mexicanum. Int J Dev Biol. 1994; 38:695700. [PubMed: 7779690]

Latacha KS, Remond MC, Ramasubramanian A, Chen AY, Elson EL, Taber LA. Role of actin polymerization in bending of the early heart tube. Dev Dyn. 2005; 233:1272-1286. [PubMed: 15986456]

Lecuit T, Lenne PF. Cell surface mechanics and the control of cell shape, tissue patterns and morphogenesis. Nat Rev Mol Cell Biol. 2007; 8:633-644. [PubMed: 17643125]

Lemanski LF. Morphology of developing heart in cardiac lethal mutant Mexican axolotls, Ambystoma mexicanum. Dev Biol. 1973; 33:312-333. [PubMed: 4789609]

Lemanski LF. Role of tropomyosin in actin filament formation in embryonic salamander heart cells. $\mathbf{J}$ Cell Biol. 1979; 82:227-238. [PubMed: 383724]

Lemanski LF, Fuldner RA, Paulson DJ. Immunofluorescence studies for myosin, alpha-actinin and tropomyosin in developing hearts of normal and cardiac lethal mutant Mexican axolotls, Ambystoma mexicanum. J Embryol Exp Morphol. 1980; 55:1-15. [PubMed: 6989943]

Littlefield R, Almenar-Queralt A, Fowler VM. Actin dynamics at pointed ends regulates thin filament length in striated muscle. Nat Cell Biol. 2001; 3:544-551. [PubMed: 11389438]

Lucitti JL, Jones EA, Huang C, Chen J, Fraser SE, Dickinson ME. Vascular remodeling of the mouse yolk sac requires hemodynamic force. Development. 2007; 134:3317-3326. [PubMed: 17720695]

Manasek FJ. Embryonic development of the heart. I. A light and electron microscopic study of myocardial development in the early chick embryo. J Morphol. 1968; 125:329-365. [PubMed: 5678904]

Manasek FJ. Determinants of heart shape in early embryos. Fed Proc. 1981; 40:2011-2016. [PubMed: 7227556]

Markwald RR. Distribution and relationship of precursor Z material to organizing myofibrillar bundles in embryonic rat and hamster ventricular myocytes. J Mol Cell Cardiol. 1973; 5:341-350. [PubMed: 4355337]

Maruyama K, Ohashi K. Tropomyosin inhibits the interaction of F-actin and filamin. J Biochem. 1978; 84:1017-1019. [PubMed: 711696]

McKeown CR, Nowak RB, Moyer J, Sussman MA, Fowler VM. Tropomodulin1 is required in the heart but not the yolk sac for mouse embryonic development. Circ Res. 2008; 103:1241-1248. [PubMed: 18927466]

McLean MD, Zajdel RW, Dube S, Thurston H, Dube DK. Tropomodulin expression in developing hearts of normal and cardiac mutant Mexican axolotl. Cardiovasc Toxicol. 2006; 6:85-98. [PubMed: 17303917]

Michele DE, Metzger JM. Physiological consequences of tropomyosin mutations associated with cardiac and skeletal myopathies. J Mol Med. 2000; 78:543-553. [PubMed: 11199327]

Moyer JD, Nowak RB, Kim NE, Larkin SK, Peters LL, Hartwig J, Kuypers FA, Fowler VM. Tropomodulin 1-null mice have a mild spherocytic elliptocytosis with appearance of tropomodulin 3 in red blood cells and disruption of the membrane skeleton. Blood. 2010; 116:2590-2599. [PubMed: 20585041]

Mudry RE, Perry CN, Richards M, Fowler VM, Gregorio CC. The interaction of tropomodulin with tropomyosin stabilizes thin filaments in cardiac myocytes. J Cell Biol. 2003; 162:1057-1068. [PubMed: 12975349]

Muthuchamy M, Pajak L, Howles P, Doetschman T, Wieczorek DF. Developmental analysis of tropomyosin gene expression in embryonic stem cells and mouse embryos. Mol Cell Biol. 1993; 13:3311-3323. [PubMed: 7684495]

Navaratnam V, Kaufman MH, Skepper JN, Barton S, Guttridge KM. Differentiation of the myocardial rudiment of mouse embryos: an ultrastructural study including freeze-fracture replication. J Anat. 1986; 146:65-85. [PubMed: 3693063]

Nishida E, Maekawa S, Sakai H. Cofilin, a protein in porcine brain that binds to actin filaments and inhibits their interactions with myosin and tropomyosin. Biochemistry. 1984; 23:5307-5313. [PubMed: 6509022] 
Nishii K, Morimoto S, Minakami R, Miyano Y, Hashizume K, Ohta M, Zhan DY, Lu QW, Shibata Y. Targeted disruption of the cardiac troponin $\mathrm{T}$ gene causes sarcomere disassembly and defects in heartbeat within the early mouse embryo. Dev Biol. 2008; 322:65-73. [PubMed: 18671960]

Nongthomba U, Clark S, Cummins M, Ansari M, Stark M, Sparrow JC. Troponin I is required for myofibrillogenesis and sarcomere formation in Drosophila flight muscle. J Cell Sci. 2004; 117:1795-1805. [PubMed: 15075240]

Nowak RB, Fischer RS, Zoltoski RK, Kuszak JR, Fowler VM. Tropomodulin1 is required for membrane skeleton organization and hexagonal geometry of fiber cells in the mouse lens. J Cell Biol. 2009; 186:915-928. [PubMed: 19752024]

Olson EN. Gene regulatory networks in the evolution and development of the heart. Science. 2006; 313:1922-1927. [PubMed: 17008524]

Ono S, Ono K. tropomyosin4 inhibits ADF/cofilin-dependent actin filament dynamics. J Cell Biol. 2002; 156:1065-1076. [PubMed: 11901171]

Otani T, Ichii T, Aono S, Takeichi M. Cdc42 GEF Tuba regulates the junctional configuration of simple epithelial cells. J Cell Biol. 2006; 175:135-146. [PubMed: 17015620]

Peckham M, Young P, Gautel M. Constitutive and variable regions of Z-disk titin/connectin in myofibril formation: a dominant-negative screen. Cell Struct Funct. 1997; 22:95-101. [PubMed: 9113395]

Ramachandran I, Terry M, Ferrari MB. Skeletal muscle myosin cross-bridge cycling is necessary for myofibrillogenesis. Cell Motil Cytoskeleton. 2003; 55:61-72. [PubMed: 12673599]

Remond MC, Fee JA, Elson EL, Taber LA. Myosin-based contraction is not necessary for cardiac clooping in the chick embryo. Anat Embryol (Berl). 2006; 211:443-454. [PubMed: 16636777]

Rethinasamy P, Muthuchamy M, Hewett T, Boivin G, Wolska BM, Evans C, Solaro RJ, Wieczorek DF. Molecular and physiological effects of alpha-tropomyosin ablation in the mouse. Circ Res. 1998; 82:116-123. [PubMed: 9440710]

Rudy DE, Yatskievych TA, Antin PB, Gregorio CC. Assembly of thick, thin, and titin filaments in chick precardiac explants. Dev Dyn. 2001; 221:61-71. [PubMed: 11357194]

Sanger JW, Ayoob JC, Chowrashi P, Zurawski D, Sanger JM. Assembly of myofibrils in cardiac muscle cells. Adv Exp Med Biol. 2000; 481:89-102. [PubMed: 10987068]

Sanger JW, Kang S, Siebrands CC, Freeman N, Du A, Wang J, Stout AL, Sanger JM. How to build a myofibril. J Muscle Res Cell Motil. 2005; 26:343-354. [PubMed: 16465476]

Schleef M, Werner K, Satzger U, Kaupmann K, Jockusch H. Chromosomal localization and genomic cloning of the mouse alpha-tropomyosin gene Tpm-1. Genomics. 1993; 17:519-521. [PubMed: 8406508]

Schoenauer R, Bertoncini P, Machaidze G, Aebi U, Perriard JC, Hegner M, Agarkova I. Myomesin is a molecular spring with adaptable elasticity. J Mol Biol. 2005; 349:367-379. [PubMed: 15890201]

Schoenauer R, Lange S, Hirschy A, Ehler E, Perriard JC, Agarkova I. Myomesin 3, a novel structural component of the M-band in striated muscle. J Mol Biol. 2008; 376:338-351. [PubMed: 18177667]

Schultheiss T, Lin ZX, Lu MH, Murray J, Fischman DA, Weber K, Masaki T, Imamura M, Holtzer H. Differential distribution of subsets of myofibrillar proteins in cardiac nonstriated and striated myofibrils. J Cell Biol. 1990; 110:1159-1172. [PubMed: 2108970]

Sehnert AJ, Huq A, Weinstein BM, Walker C, Fishman M, Stainier DY. Cardiac troponin T is essential in sarcomere assembly and cardiac contractility. Nat Genet. 2002; 31:106-110. [PubMed: 11967535]

Sharp WW, Simpson DG, Borg TK, Samarel AM, Terracio L. Mechanical forces regulate focal adhesion and costamere assembly in cardiac myocytes. Am J Physiol. 1997; 273:H546-556. [PubMed: 9277468]

Sharp WW, Terracio L, Borg TK, Samarel AM. Contractile activity modulates actin synthesis and turnover in cultured neonatal rat heart cells. Circ Res. 1993; 73:172-183. [PubMed: 8508528]

Shiraishi I, Takamatsu T, Fujita S. 3-D observation of N-cadherin expression during cardiac myofibrillogenesis of the chick embryo using a confocal laser scanning microscope. Anat Embryol (Berl). 1993; 187:115-120. [PubMed: 8238958] 
Shiraishi I, Takamatsu T, Minamikawa T, Fujita S. 3-D observation of actin filaments during cardiac myofibrinogenesis in chick embryo using a confocal laser scanning microscope. Anat Embryol (Berl). 1992; 185:401-408. [PubMed: 1609966]

Soeno Y, Shimada Y, Obinata T. BDM (2,3-butanedione monoxime), an inhibitor of myosin-actin interaction, suppresses myofibrillogenesis in skeletal muscle cells in culture. Cell Tissue Res. 1999; 295:307-316. [PubMed: 9931377]

Starr CM, Diaz JG, Lemanski LF. Analysis of actin and tropomyosin in hearts of cardiac mutant axolotls by two-dimensional gel electrophoresis, western blots, and immunofluorescent microscopy. J Morphol. 1989; 201:1-10. [PubMed: 2664186]

Stehn JR, Schevzov G, O’Neill GM, Gunning PW. Specialisation of the tropomyosin composition of actin filaments provides new potential targets for chemotherapy. Curr Cancer Drug Targets. 2006; 6:245-256. [PubMed: 16712460]

Stromer MH, Goll DE. Studies on purified alpha-actinin. II. Electron microscopic studies on the competitive binding of alpha-actinin and tropomyosin to Z-line extracted myofibrils. J Mol Biol. 1972; 67:489-494. [PubMed: 5045309]

Szczesna-Cordary D, Morimoto S, Gomes AV, Moore JR. Cardiomyopathies: classification, clinical characterization, and functional phenotypes. Biochem Res Int. 2012; 2012:870942. [PubMed: 23304510]

Temm-Grove CJ, Jockusch BM, Weinberger RP, Schevzov G, Helfman DM. Distinct localizations of tropomyosin isoforms in LLC-PK1 epithelial cells suggests specialized function at cell-cell adhesions. Cell Motil Cytoskeleton. 1998; 40:393-407. [PubMed: 9712268]

Turnacioglu KK, Mittal B, Dabiri GA, Sanger JM, Sanger JW. Zeugmatin is part of the Z- band targeting region of titin. Cell Struct Funct. 1997; 22:73-82. [PubMed: 9113393]

Wang SM, Greaser ML, Schultz E, Bulinski JC, Lin JJ, Lessard JL. Studies on cardiac myofibrillogenesis with antibodies to titin, actin, tropomyosin, and myosin. J Cell Biol. 1988; 107:1075-1083. [PubMed: 3047149]

Weber A, Pennise CR, Babcock GG, Fowler VM. Tropomodulin caps the pointed ends of actin filaments. J Cell Biol. 1994; 127:1627-1635. [PubMed: 7798317]

Weber A, Pennise CR, Fowler VM. Tropomodulin increases the critical concentration of barbed endcapped actin filaments by converting ADP.P(i)-actin to ADP-actin at all pointed filament ends. J Biol Chem. 1999; 274:34637-34645. [PubMed: 10574928]

Weber KL, Fischer RS, Fowler VM. Tmod3 regulates polarized epithelial cell morphology. J Cell Sci. 2007; 120:3625-3632. [PubMed: 17928307]

Wegner A. Equilibrium of the actin-tropomyosin interaction. J Mol Biol. 1979; 131:839-853. [PubMed: 513132]

Wegner A, Walsh TP. Interaction of tropomyosin-troponin with actin filaments. Biochemistry. 1981; 20:5633-5642. [PubMed: 6457636]

Weigt C, Schoepper B, Wegner A. Tropomyosin-troponin complex stabilizes the pointed ends of actin filaments against polymerization and depolymerization. FEBS Lett. 1990; 260:266-268. [PubMed: 2298302]

Yamashiro S, Gokhin DS, Kimura S, Nowak RB, Fowler VM. Tropomodulins: Pointed-end capping proteins that regulate actin filament architecture in diverse cell types. Cytoskeleton. 2012; 69:337-370. [PubMed: 22488942]

Yang YZ, Korn ED, Eisenberg E. Cooperative binding of tropomyosin to muscle and Acanthamoeba actin. J Biol Chem. 1979; 254:7137-7140. [PubMed: 156726]

Yu JG, Russell B. Cardiomyocyte remodeling and sarcomere addition after uniaxial static strain in vitro. J Histochem Cytochem. 2005; 53:839-844. [PubMed: 15995142]

Zajdel RW, Dube DK, Lemanski LF. The cardiac mutant Mexican axolotl is a unique animal model for evaluation of cardiac myofibrillogenesis. Exp Cell Res. 1999; 248:557-566. [PubMed: 10222147]

Zajdel RW, McLean MD, Lemanski SL, Muthuchamy M, Wieczorek DF, Lemanski LF, Dube DK. Ectopic expression of tropomyosin promotes myofibrillogenesis in mutant axolotl hearts. Dev Dyn. 1998; 213:412-420. [PubMed: 9853962] 
Zajdel RW, Thurston H, Prayaga S, Dube S, Poiesz BJ, Dube DK. A reduction of tropomyosin limits development of sarcomeric structures in cardiac mutant hearts of the Mexican axolotl. Cardiovasc Toxicol. 2007; 7:235-246. [PubMed: 17990128]

Zeece MG, Robson RM, Bechtel PJ. Interaction of alpha-actinin, filamin and tropomyosin with Factin. Biochim Biophys Acta. 1979; 581:365-370. [PubMed: 518922]

Zhao L, Zhao X, Tian T, Lu Q, Skrbo-Larssen N, Wu D, Kuang Z, Zheng X, Han Y, Yang S, Zhang C, Meng A. Heart-specific isoform of tropomyosin4 is essential for heartbeat in zebrafish embryos. Cardiovasc Res. 2008; 80:200-208. [PubMed: 18583338] 


\section{Key findings}

1. A function for tropomyosin (TM) in mammalian myofibril assembly and cardiac development was explored via a deletion in the mouse TPM1 gene targeting aTM1, the major striated muscle TM isoform.

2. Mice lacking aTM1 are embryonic lethal at E9.5 due to aberrant myofibril assembly and/or degeneration of partially assembled sarcomeres, leading to a grossly enlarged, misshapen, and non-beating heart with an abnormally thin myocardium and reduced trabeculae.

3. Failure of a-actinin/F-actin adherens belt assembly in both aTM1- and Tmod1deficient cardiomyocytes identifies a novel aTM1/Tmod1-based pathway stabilizing F-actin at cell-cell junctions. 


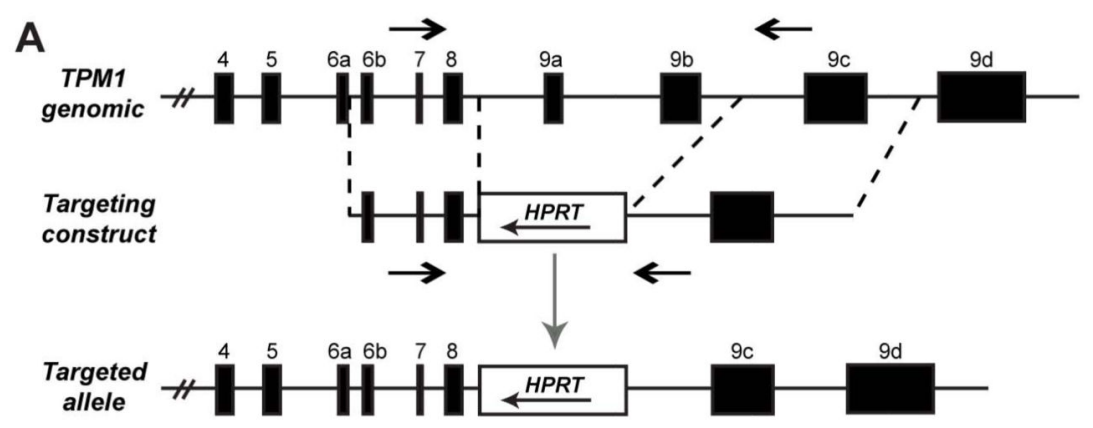

B
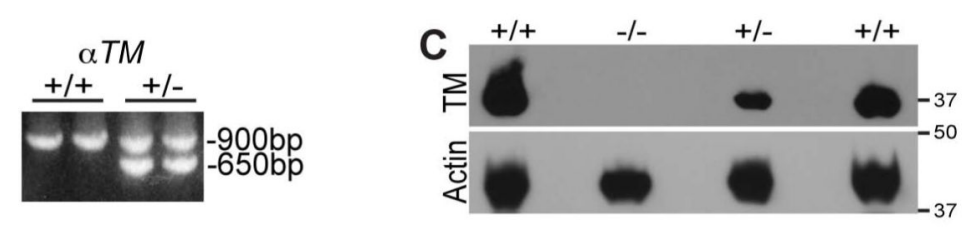

Figure 1. a TM1 $^{-/-}$embryos do not express a TM1

(A) Schematic of the wild-type and targeted aTM1 (TPM1) gene locus, indicating replacement of exons $9 \mathrm{a}$ and $9 \mathrm{~b}$ by the HPRT gene as described in Figure 1 from

(Rethinasamy et al., 1998), modified with current TPM exon nomenclature (Gunning et al., 2008). Forward and reverse primers for wild-type and targeted alleles are indicated (see Materials and Methods). (B) PCR genotyping of E8.5 yolk sac DNA from representative wild-type (+/+) and heterozygous (+/-) embryos. (C) Western blots of E8.5 whole embryos generated from an $\mathrm{aTM1}^{+/-}$intercross. Blots were probed with the $\mathrm{CH} 1$ anti-TM antibody, recognizing exon 9a of aTM1 (top). A pan-actin antibody was used as a loading control (bottom). 

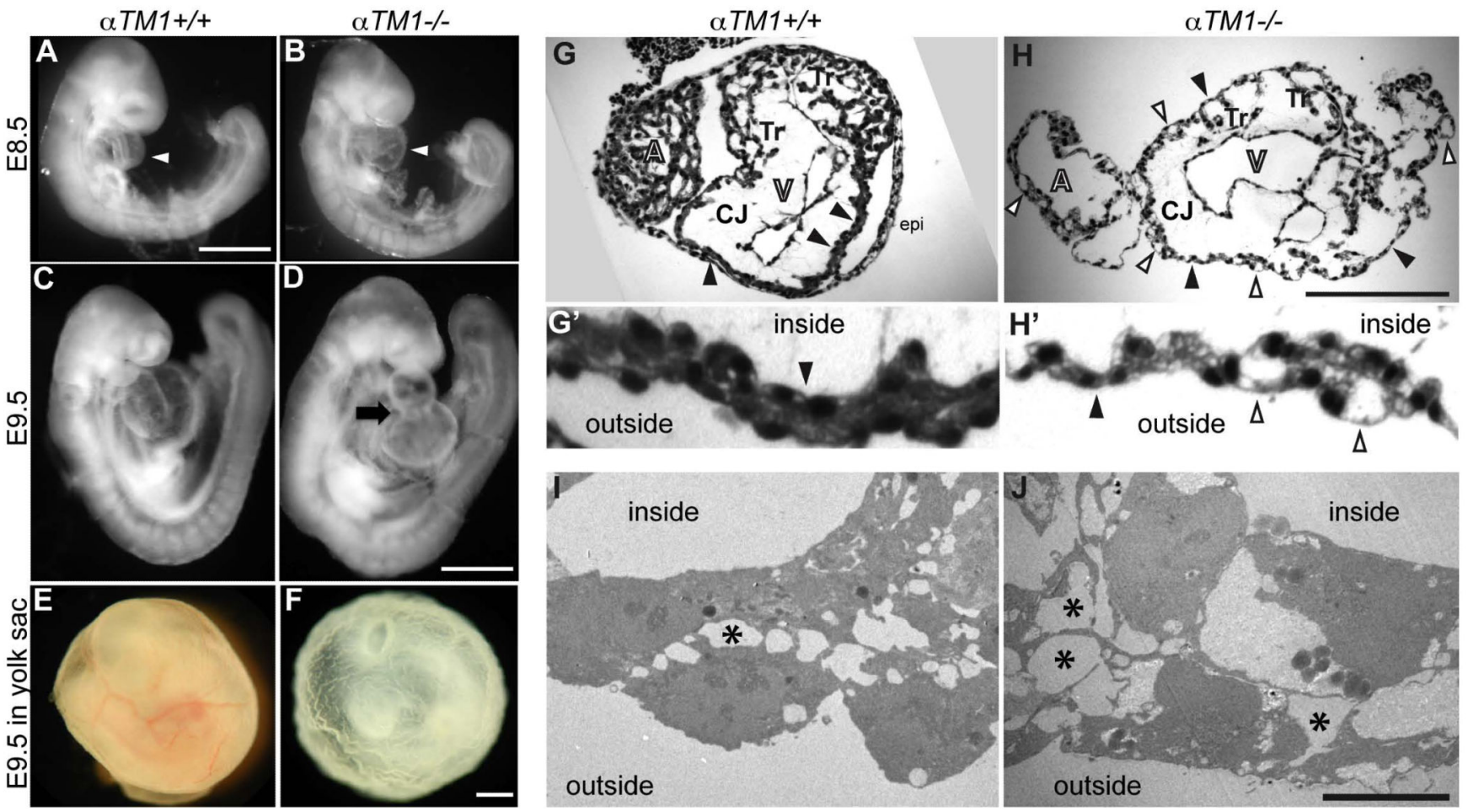

Figure 2. aTM1 ${ }^{-/-}$embryos have dysmorphic and enlarged hearts at E8.5-9.5, with an abnormally thin and compact myocardium with reduced trabeculae

(A-D) Gross morphology of (A,C) a $T M 1^{+/+}$and (B,D) a $T M 1^{-/-}$embryos (side view) at

E8.5 (12 somite pairs) and E9.5 (17 somite pairs). Arrowheads in A,B indicate the location of the E8.5 heart; black arrow in D points to the abnormal constriction in the outflow tract in the aTM1-/- E9.5 embryo. (E,F) Gross morphology of the E9.5 yolk sac. Note that the vasculature present in the a TM1 $1^{+/+}$yolk sac $(\mathrm{E})$ is completely absent in the a $T M 1^{-/-}$yolk sac (F). Scale bars: $500 \mu \mathrm{m}$ in A-F. (G,H) Histology of E9.5 hearts from aTM1+/+ $\left(\mathrm{G}_{,} \mathrm{G}^{\prime}, 22\right.$ somite pairs) and a $T M 1^{-/-}\left(\mathrm{H}, \mathrm{H}^{\prime}, 22\right.$ somite pairs $)$ embryos. The a $T M 1^{-/-}$heart appears larger and dilated, due to thinning of the compact myocardium and a reduction in trabeculae. Black arrowheads in $\mathrm{G}, \mathrm{G}^{\prime}$ point to the compact myocardium; black arrowheads in $\mathrm{H}, \mathrm{H}^{\prime}$ indicate regions where the myocardium is only one cell layer thick in the a $T M 1^{-/-}$heart; open arrowheads in $\mathrm{H}, \mathrm{H}^{\prime}$ indicate intercellular spaces in the a $T M 1^{-/-}$myocardium. In $\mathrm{H}$, the epicardium of the a TM1 $1^{-/-}$heart was torn and removed during dissection. (I, J) TEM of myocardium from aTM1 ${ }^{+/+}$and $\mathrm{aTM1} 1^{-/-}$hearts. Asterisks indicate intercellular spaces, which are greatly expanded in the $a T M 1^{-/-}$heart. Abbreviations: A, atrium; V, ventricle; CJ, cardiac jelly; epi, epicardium; Tr, trabeculae. Scale bars: $200 \mu \mathrm{m}$ in $\mathrm{G}, \mathrm{H} ; 20 \mu \mathrm{m}$ in $\mathrm{G}^{\prime}, \mathrm{H}$

'. Scale bars: $10 \mu \mathrm{m}$ in I, J. 

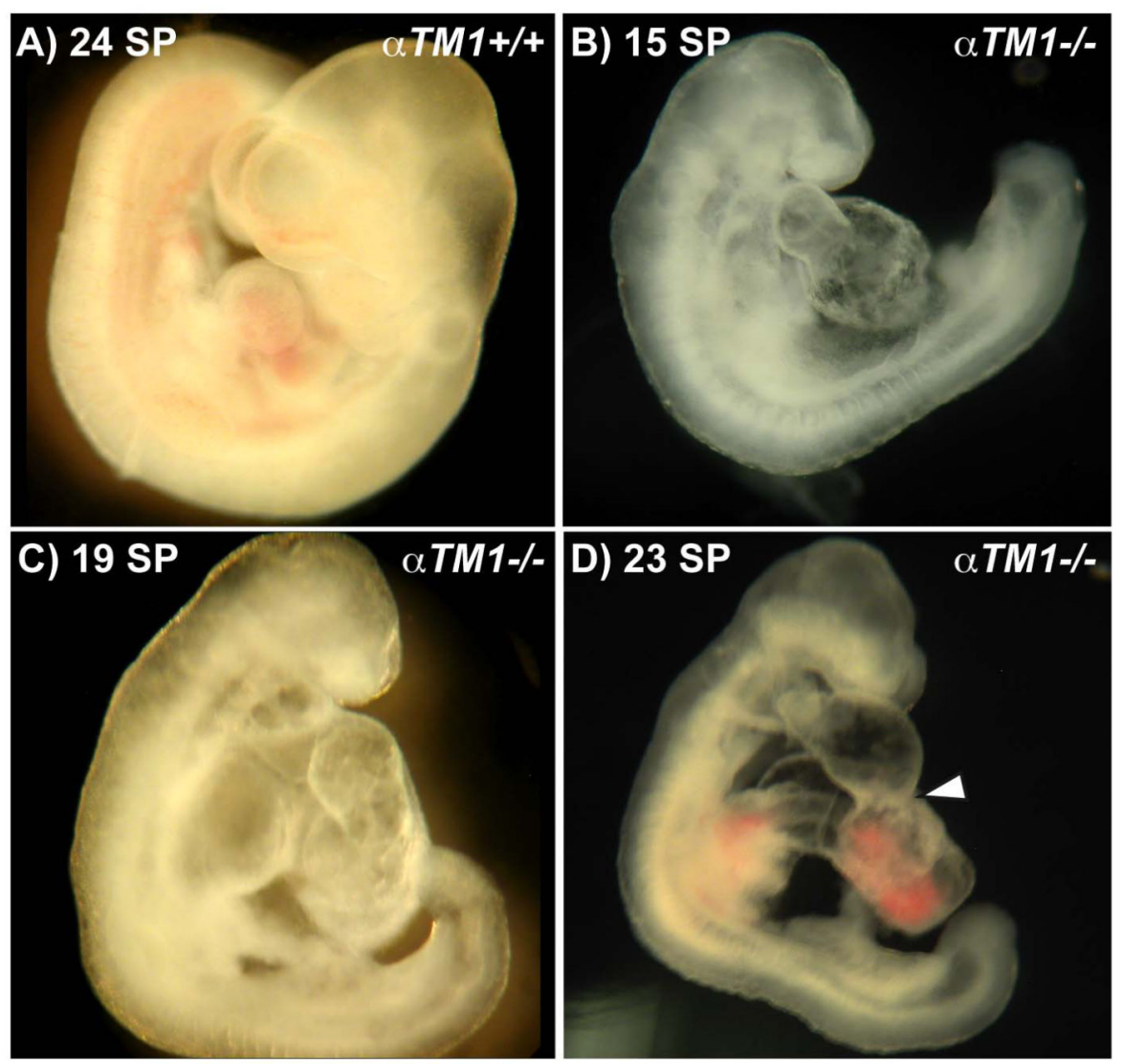

Figure 3. a MMI $^{-/-}$embryos have highly enlarged, irregularly shaped hearts at E9.5 Gross morphology of E9.5 (A) a TM1 ${ }^{+/+}$(24 somite pairs) and (B-D) a $T M 1^{-/-}$embryos (B, 15 somite pairs; C, 19 somite pairs; D, 23 somite pairs). An abnormal constriction of the outflow tract(white arrowhead) is seen in the $a T M 1^{-/-}$embryos. Also note the wrinkled appearance of the a $T M 1^{-/-}$heart tube evident in B, and the extremely enlarged and dysmorphic a $T M 1^{-/-}$heart in D. 

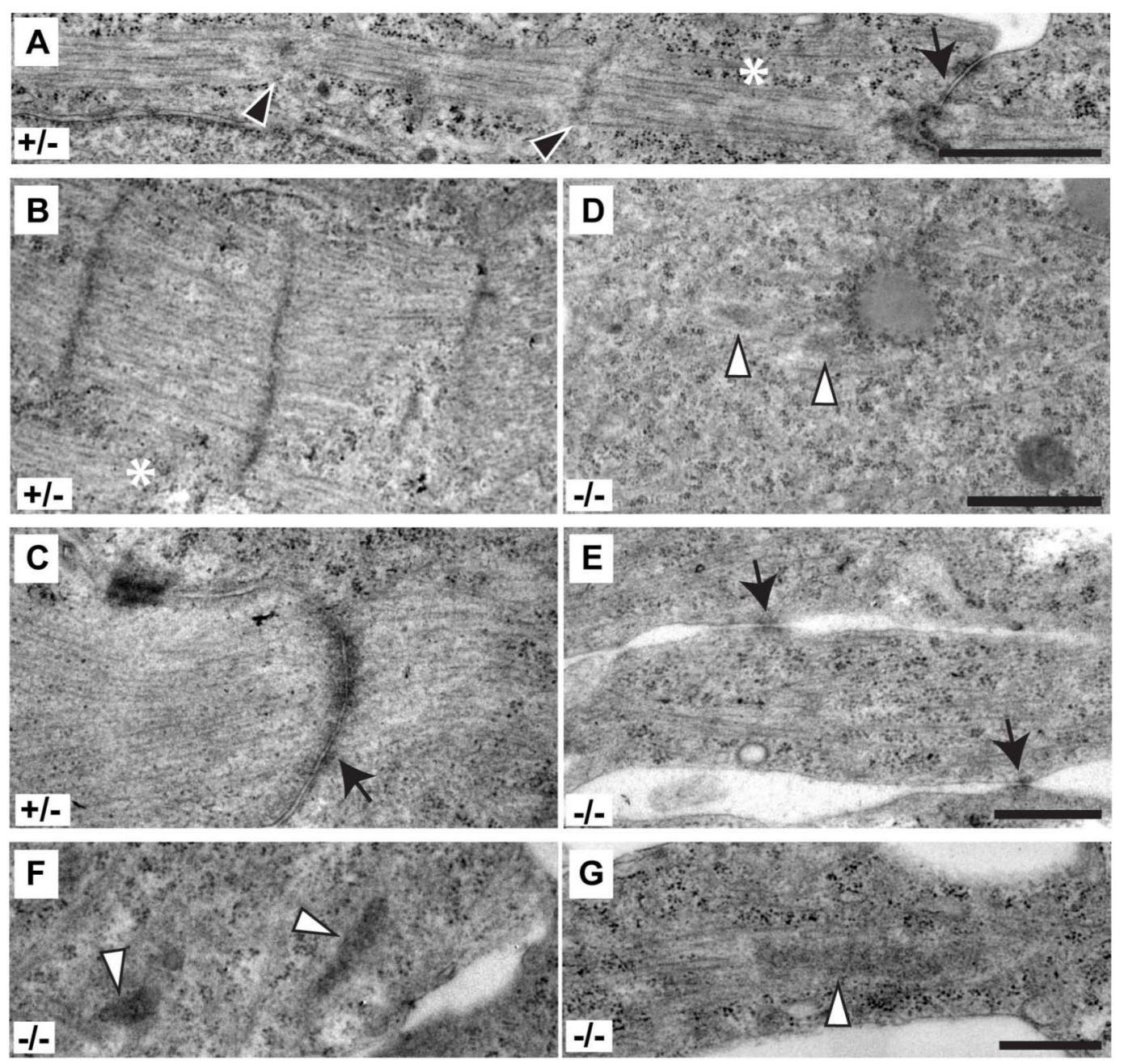

Figure 4. TEM reveals failure of myofibril assembly and adherens junction formation in aTM1 $^{-/-}$hearts at E8.5

(A-C) $a T M 1^{+/-}$and (D-G) a TM1 ${ }^{-/-}$embryos. (A) a $T M 1^{+/-}$cardiomyocyte with a typical striated myofibril inserting at one end into an adherens junction at the membrane. (B)

Mature sarcomeres in a $T M 1^{+/-}$cardiomyocytes. (C) A large, electron-dense adherens junction with myofibrils at the membrane connecting adjacent $a T M M^{+/-}$cardiomyocytes.

(D) $\mathrm{aTM1}^{-/-}$cardiomyocyte containing Z-bodies and disorganized thin and thick filaments.

(E) $\mathrm{aTM1}^{-/-}$cardiomyocytes with small adherens junctions without attached myofibrils.

Scattered thick filaments but no clear Z-bodies are present in the cytoplasm in this region. (F, G) Large, electron-dense Z-bodies resembling the rods seen only in a $\mathrm{TMI}^{-/-}$ cardiomyocytes. Black arrowheads, Z-lines; white asterisks, H-zones with M-lines; black arrows, adherens junctions; white arrowheads, Z-bodies. Scale bars: (A, B, D) $1 \mu \mathrm{m}$; (C, E, F, G) $500 \mathrm{~nm}$. 

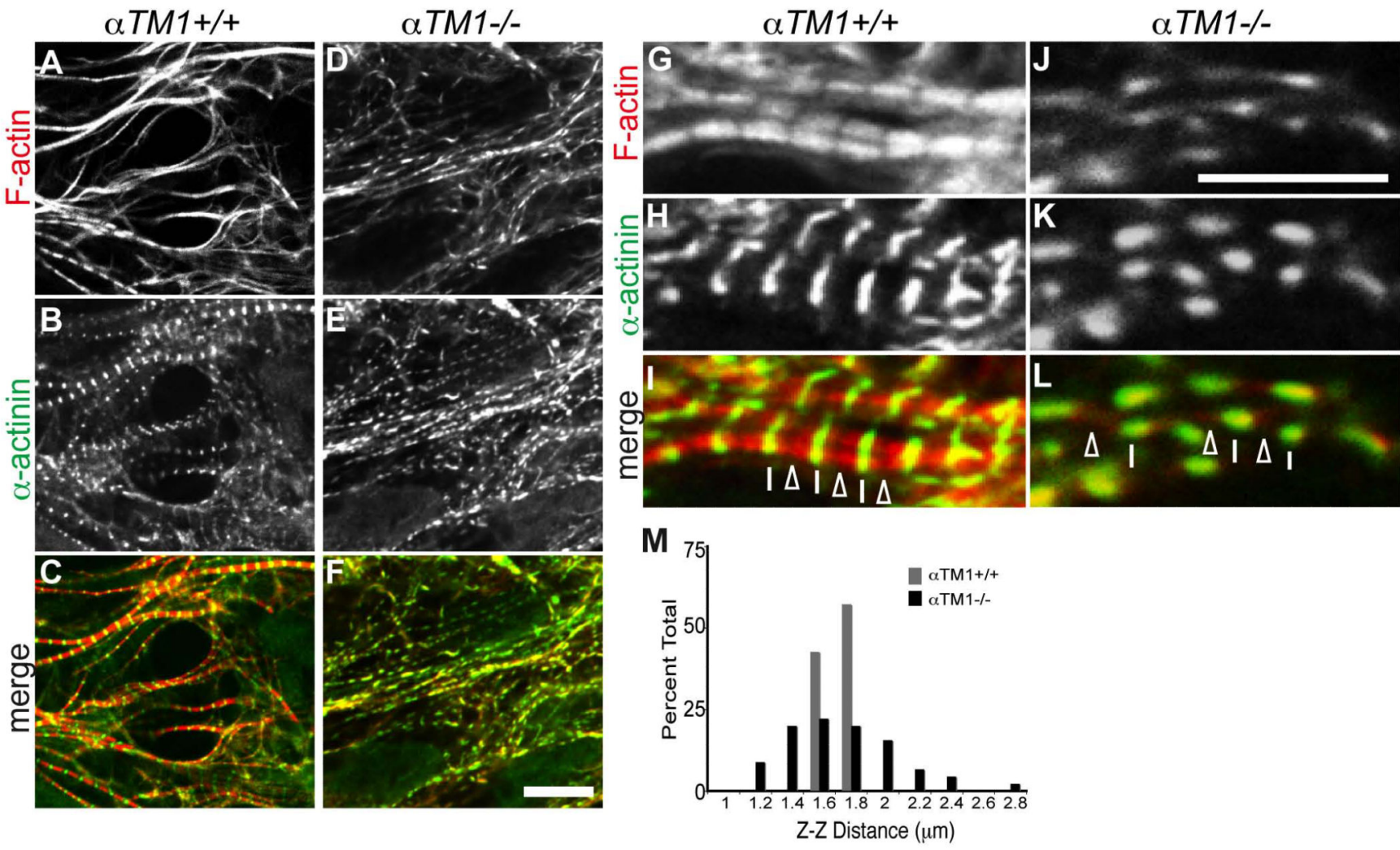

Figure 5. Sarcomeric a-actinin assembles into irregular puncta along aberrant $\mathbf{F}$-actin fibrils in the aTM1 $^{-1-}$ embryonic mouse heart

Immunofluorescence staining of sarcomeric a-actinin and F-actin in cardiomyocytes in the outer wall of the heart in (A-C, G-I) aTM1 ${ }^{+/+}$and (DF, J-L) aTM1 ${ }^{-/-}$E8.5 mouse embryos. Sarcomeric a-actinin (B,E,H,K, green in merges C,F,I,L) and F-actin (A,D,G,J, red in merges $\mathrm{C}, \mathrm{F}, \mathrm{I}, \mathrm{L})$ are organized in typical striated myofibrils with periodic Z-lines and gaps in F-actin (H-zones) in a TMI ${ }^{+/+}$embryos, but not in a $T M 1^{-/-}$embryos, which instead show irregular a-actinin puncta along F-actin fibrils. I, in a $T M 1^{+/+}$cardiomyocytes, open arrowheads indicate $\mathrm{H}$-zones and lines indicate $\mathrm{Z}$-lines. $\mathrm{L}$, in a $T M 1^{-/}$- cardiomyocytes, lines indicate aberrant $\mathbf{a}$-actinin puncta (Z-bodies) and open arrowheads indicate positions equidistant between adjacent $\mathrm{a}$-actinin puncta where the $\mathrm{H}$-zone should reside, but does not.

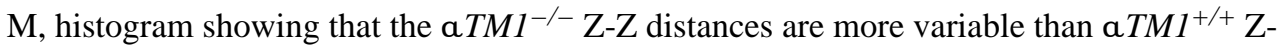
$\mathrm{Z}$ distances (F-test; $p=2.84 \times 10^{-25}$ ). Scale bars: (A-F) $9 \mu \mathrm{m}$; (G-L) $5 \mu \mathrm{m}$. 
titin Z1Z2
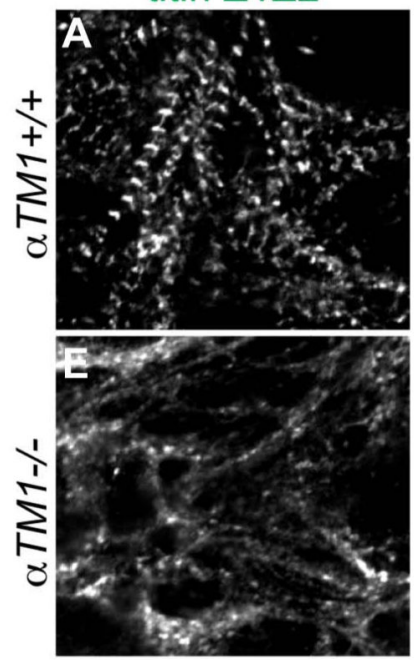

F-actin
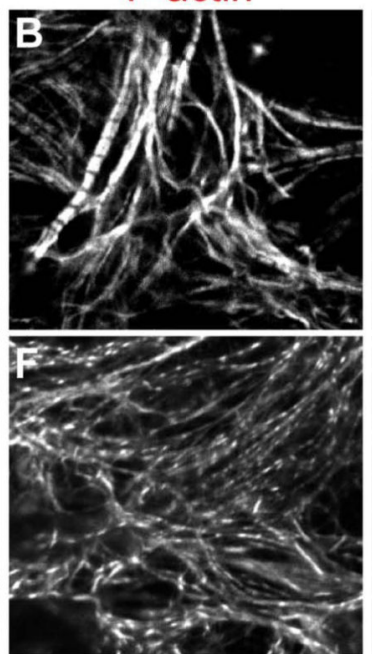

$\alpha$-actinin

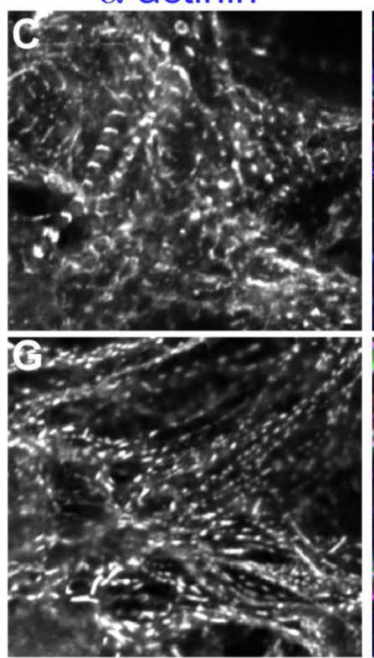

merge
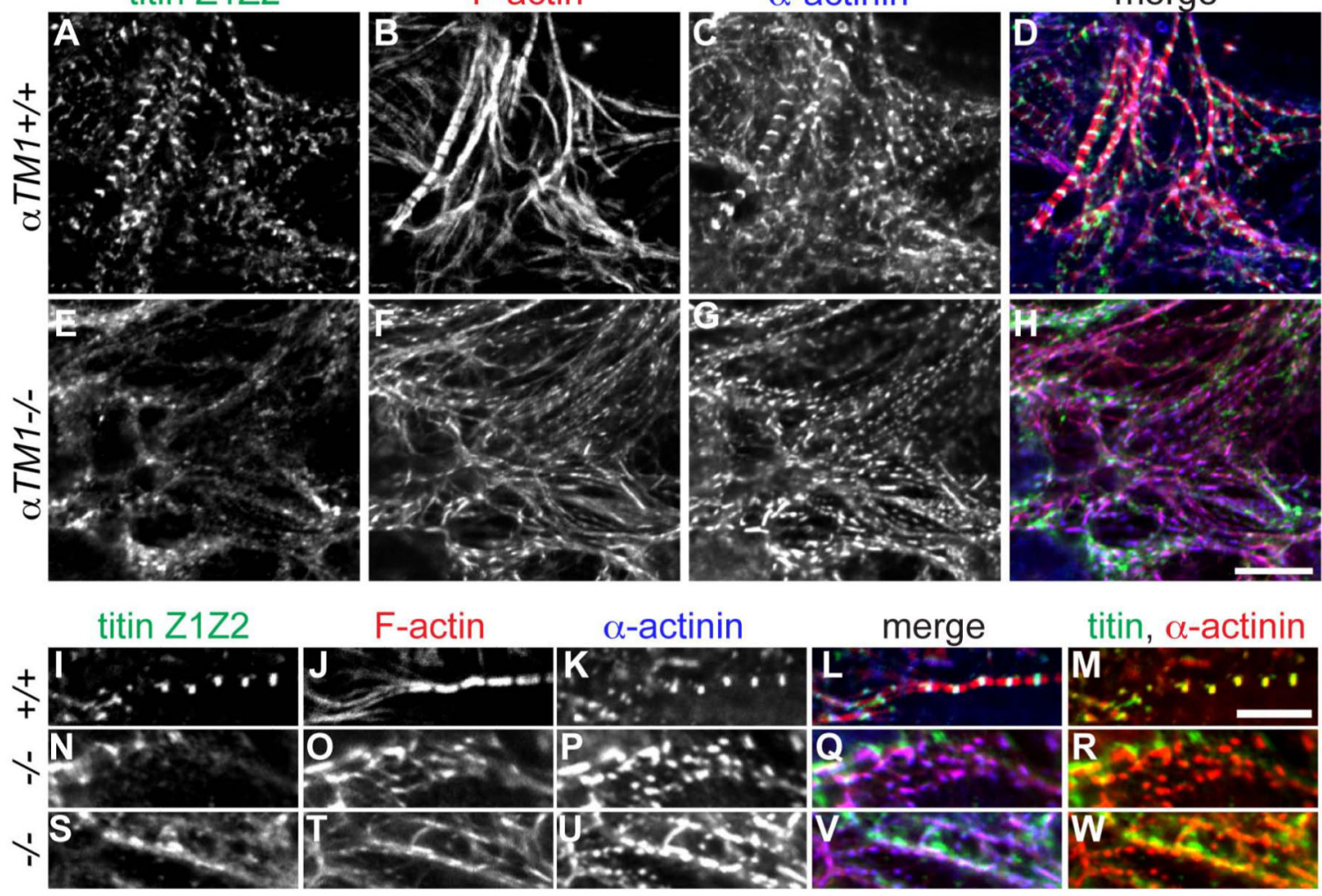

Figure 6. Assembly of the titin $\mathrm{Z1Z2}$ epitope is not well coordinated with sarcomeric a-actinin in aTM1 ${ }^{-1-}$ cardiomyocytes

Immunofluorescence staining of titin Z1Z2, F-actin, and sarcomeric a-actinin in cardiomyocytes in E8.5 a TM1 ${ }^{+/+}$(A-D) and a TM1 ${ }^{-/-}$(E-H) embryos. Titin Z1Z2 (A,E; green in merges $\mathrm{D}, \mathrm{H}), \mathrm{F}$-actin $(\mathrm{B}, \mathrm{F}$; red in merges $\mathrm{D}, \mathrm{H})$, and a-actinin $(\mathrm{C}, \mathrm{G}$; blue in merges $\mathrm{D}, \mathrm{H})$. High-magnification views show that (I-M) titin Z1Z2 and a-actinin are at the Z-lines of an $\mathrm{aTMI}^{+/+}$striated myofibril, while (N-W) titin Z1Z2 is inconsistently associated with a-actinin puncta in the aTM1 ${ }^{-/}$F-actin fibrils. Scale bars: (A-H) $9 \mu \mathrm{m}$; (I-R) $6 \mu \mathrm{m}$. 

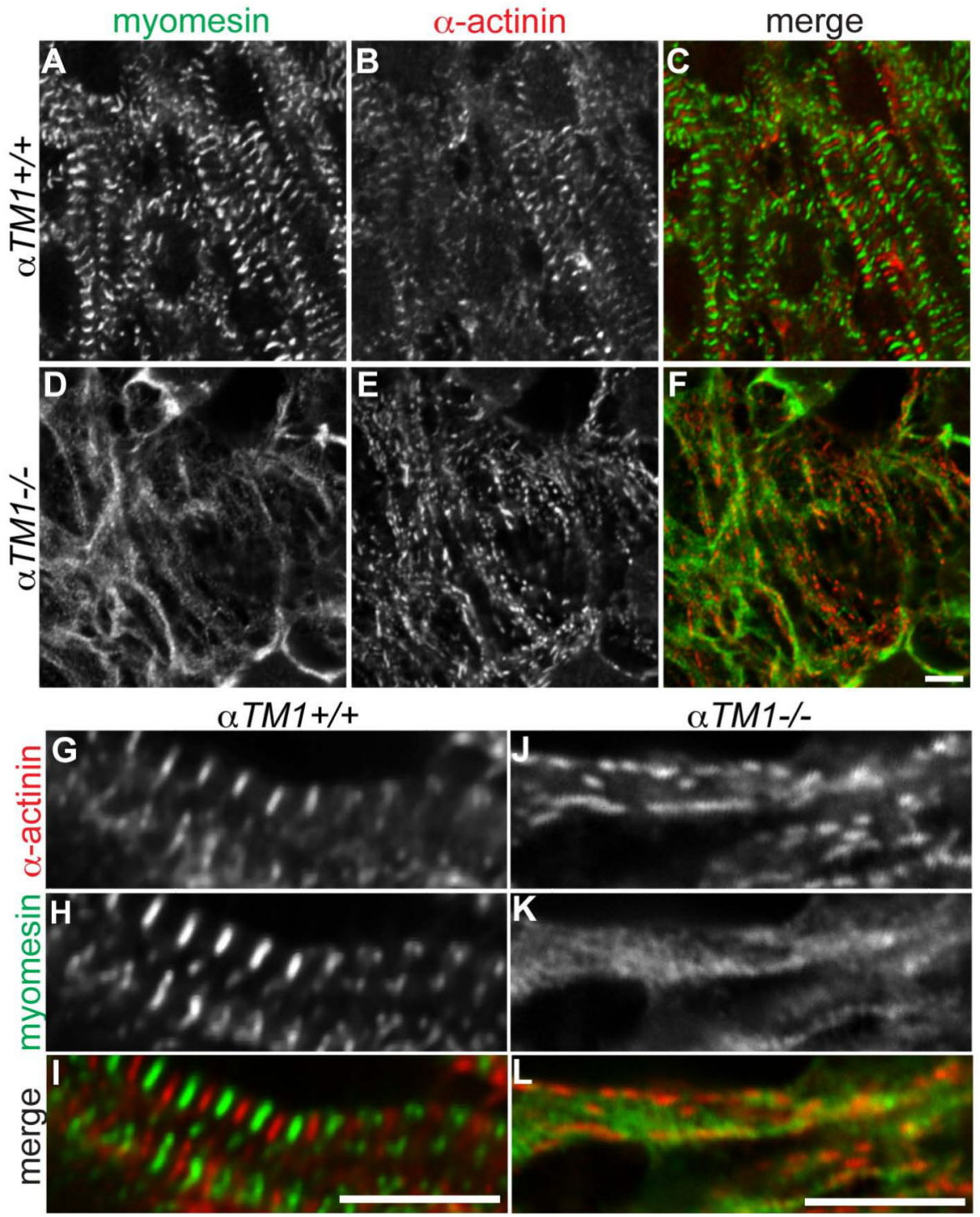

Figure 7. Myomesin accumulates near $a$-actinin/F-actin fibrils but fails to assemble in a periodic pattern in a $\mathrm{TMI}^{-/-}$cardiomyocytes

Immunofluorescence staining of myomesin (A,D,H,I: green in merges C,F,I,L) and sarcomeric a-actinin (B,E,G,J: red in merges $\mathrm{C}, \mathrm{F}, \mathrm{I}, \mathrm{L})$ in cardiomyocytes of E8.5 aTMI+/+ (A-C, G-I) and aTM1-/- (D-F, J-L) embryos. In a $T M 1^{+/+}$myofibrils, myomesin is at the $\mathrm{M}$-line alternating with sarcomeric $\mathrm{a}$-actinin at the $\mathrm{Z}$-line, as expected from normal thick filament organization in sarcomeres. In a $T M 1^{-/-}$myofibrils, myomesin accumulates in disorganized clumps near the F-actin fibrils with a-actinin puncta, but is not organized in a striated or periodic pattern. Scale bars: (A-F) $4 \mu \mathrm{m}$; (G-L) $6 \mu \mathrm{m}$. 

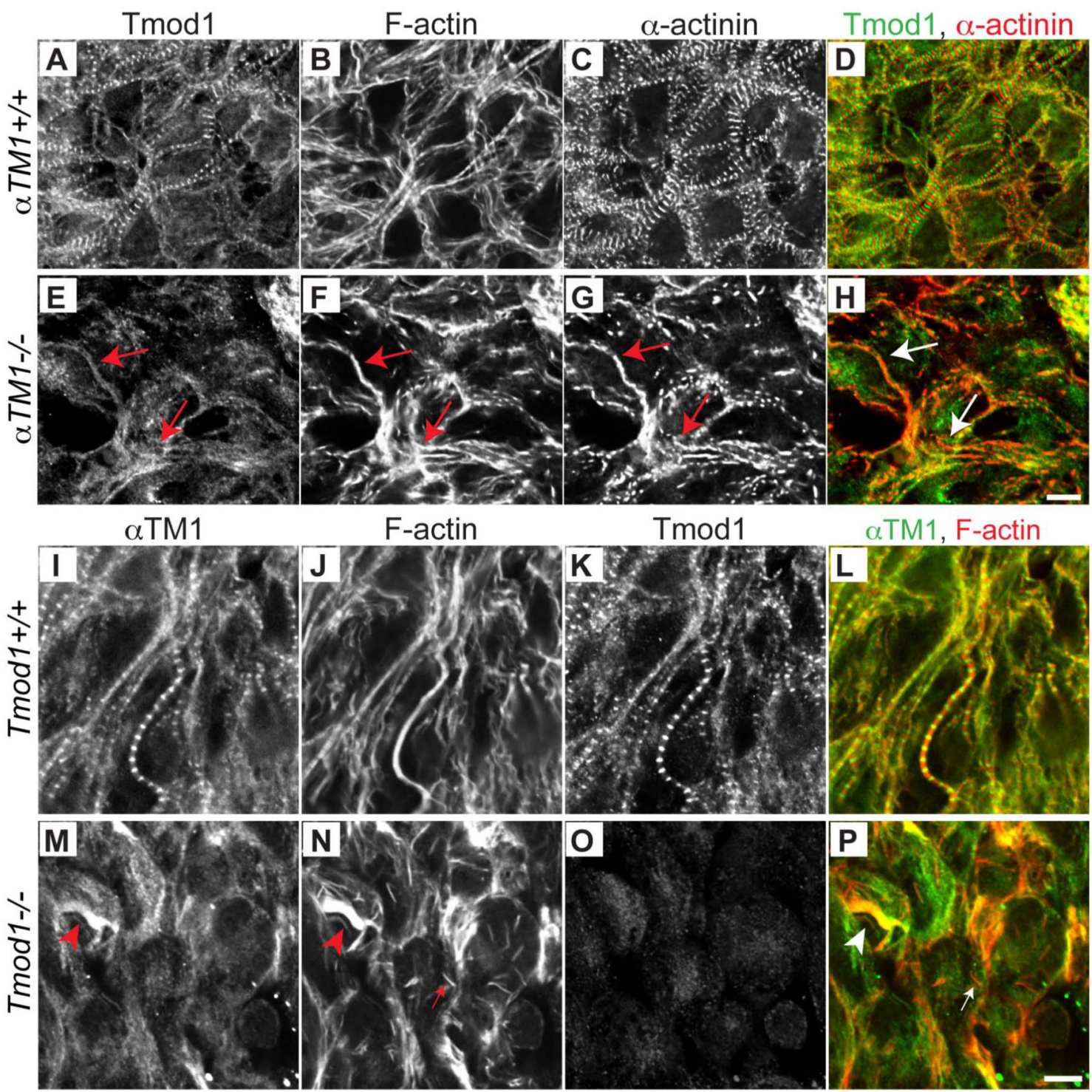

Figure 8. Tmod1 and a TM1 do not assemble into a-actinin/F-actin fibrils in a $\mathrm{TMI}^{-/-}$and Tmod1 ${ }^{-1-}$ cardiomyocytes, respectively

(A-H) Tmod1, F-actin, and sarcomeric a-actinin assembly in cardiomyocytes in the outer wall of the E8.5 heart of (A-D) aTM1 ${ }^{+/+}$and (E-H) aTM1 ${ }^{-/-}$mouse embryos.

Immunofluorescence staining of Tmod1 (A,E; green in merges D,H), F-actin $(\mathrm{B}, \mathrm{F})$ and aactinin $(\mathrm{C}, \mathrm{G}$; red in merges $\mathrm{D}, \mathrm{H})$ are shown. a $T M 1^{+/+}$cardiomyocytes show periodic

Tmod1 stripes at gaps in F-actin (H-zones) alternating with a-actinin stripes at Z-lines in typical striated myofibrils. In a TM1 $1^{-/}$embryos, Tmod 1 accumulates in the vicinity of aactinin/F-actin fibrillar structures, but is not organized in a periodic pattern. Red or white arrows, nonstriated sarcomeric a-actinin/F-actin fibrils containing diffuse Tmod1. (I-P) aTM1, F-actin, and Tmod1 assembly in cardiomyocytes in the outer wall of the E8.5 heart in (I-L) Tmod1 ${ }^{+/+}$and (MP) Tmod1 ${ }^{-/-}$mouse embryos. Immunofluorescence staining of aTM1 (I,M; green in merges L,P), F-actin (J,N; red in merges L,P) and Tmod1 $(\mathrm{K}, \mathrm{O})$ are shown. Tmod1 ${ }^{+/+}$cardiomyocytes show typical striated myofibrils with periodic aTM1 
bands. In $\mathrm{Tmod1}^{-1-}$ cardiomyocytes, diffuse aggregates of aTM1 partially co-localize with large F-actin aggregates. Red or white arrowheads, large F-actin bundles or aggregates with Tmod1 and aTM1; red or white arrows, small F-actin rod lacking Tmod1 and aTM1. Scale bars: $6 \mu \mathrm{m}$. 

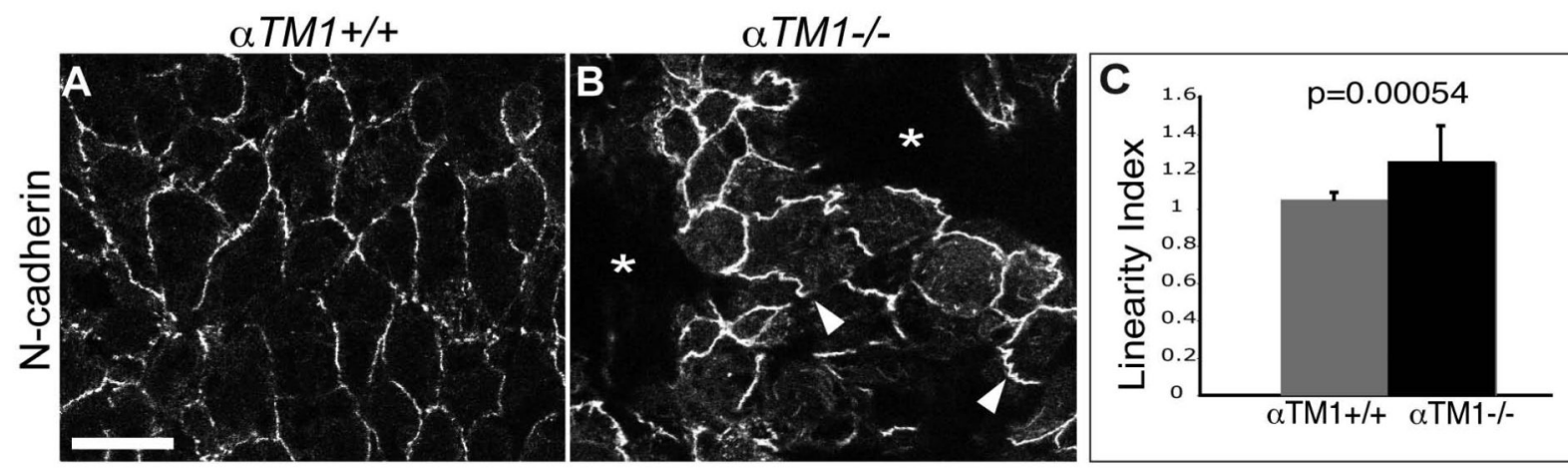

Figure 9. N-cadherin-containing cell-cell junctions display increased tortuosity in cardiomyocytes from a $\mathrm{TM1}^{-1-}$ embryonic mouse hearts

E8.5 hearts from (A) aTM1 ${ }^{+/+}$and (B) aTM1 ${ }^{-/-}$embryos were immunostained for $\mathrm{N}$ cadherin. Only the outermost optical section of cardiomyocytes in the heart wall, containing the apical surface and cell junctions of the cardiomyocytes, is shown. White arrowheads in $\mathrm{B}$ indicate tortuous cell boundaries in a $\mathrm{TM1}^{-/-}$cardiomyocytes, while asterisks indicate regions where the myocardial sheet is absent from the optical section due to infolding of the myocardium. Panel $\mathrm{C}$ is a bar graph depicting the linearity indices of $\mathrm{N}$-cadherin immunostaining in aTM1 ${ }^{+/+}(n=35)$ and aTM1 ${ }^{-/-}(n=32)$ hearts; error bars indicate SD. Scale bar: $6 \mu \mathrm{m}$. 

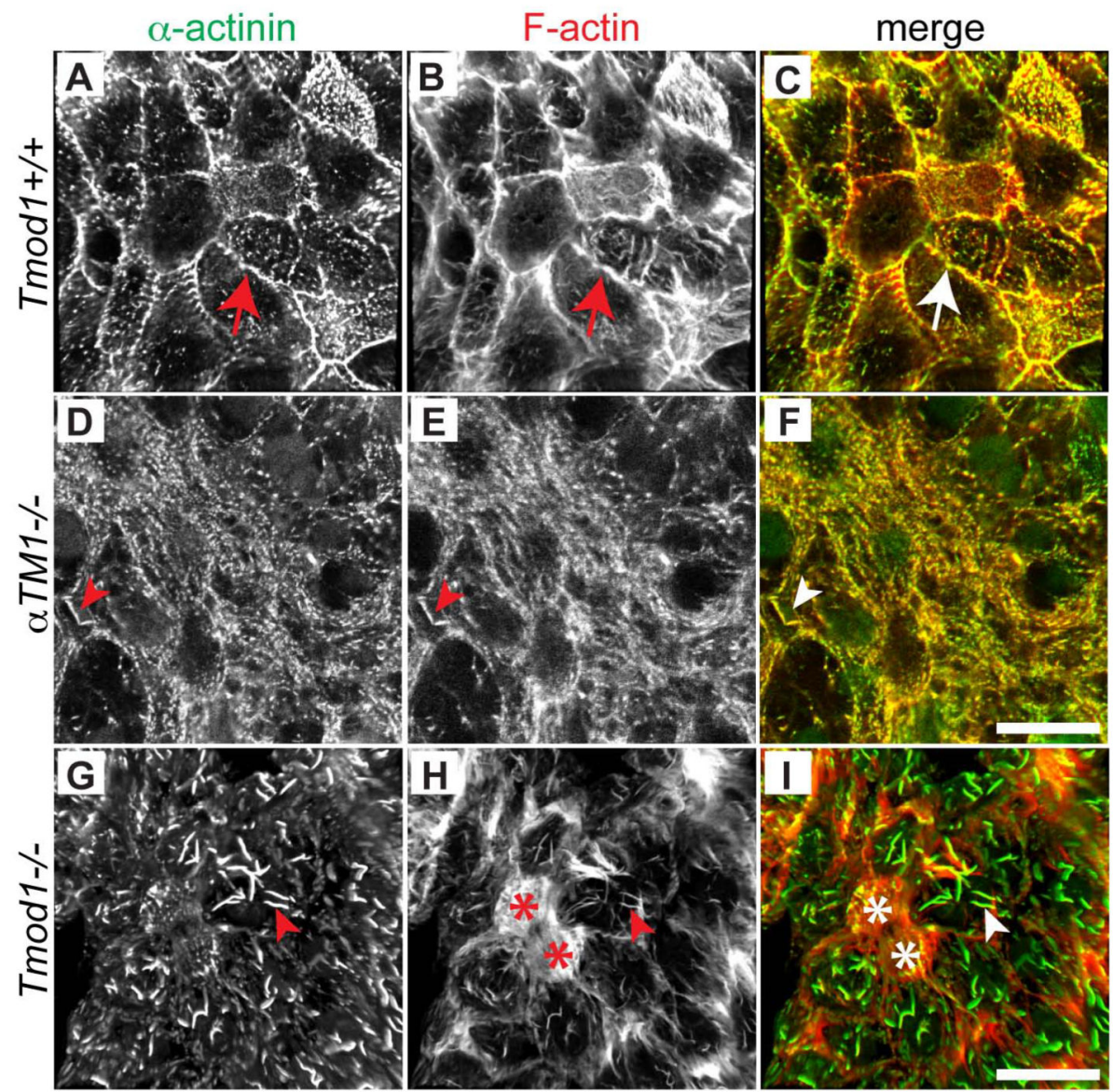

Figure 10. $a$-actinin/F-actin adherens belts fail to assemble at apical cell junctions in cardiomyocytes in aTM1 ${ }^{-/-}$and Tmod1 $^{-1-}$ embryonic mouse hearts

Immunofluorescence of cardiomyocytes in the outer wall of E8.5 hearts from wild-type (AC), a TM1-/- (D-F), and Tmod1 ${ }^{-/-}(\mathrm{G}-\mathrm{I})$ mouse embryos, stained for sarcomeric a-actinin (A,D,G; green in merges $\mathrm{C}, \mathrm{F}, \mathrm{I})$ and F-actin (B,E,H; red in merges C,F,I). Only the outermost $2 \mu \mathrm{m}$ of the outer layer of cardiomyocytes in the heart walls are shown, containing their apical surface (3 optical sections). In wild-type cardiomyocytes (A-C), sarcomeric a-actinin and F-actin form continuous circumferential belts (red and white arrows) near the apical ends of the cells along the outer surface of the heart. In aTM1-/- and Tmod1 ${ }^{-/}$cardiomyocytes, sarcomeric a-actinin and F-actin do not assemble in circumferential belts at the cell-cell junctions. Instead, in a $T M 1^{-/-}$cardiomyocytes (D-F), a-actinin assembles into puncta and occasional rods associated with disorganized F-actin fibrils in the cytoplasm (red and white arrowheads). In Tmod1 $1^{-/-}$cardiomyocytes (G-I), aactinin predominantly assembles into rods that stain weakly for F-actin (red and white arrowheads), and F-actin accumulates in large bundles and aggregates in the cytoplasm (red and white asterisks). Scale bars: $19 \mu \mathrm{m}$. 
Table 1

Litter analysis of aTM1 ${ }^{+/-} \times \mathrm{aTM1}^{+/-}$progeny reveals that aTM1 $1^{-/-}$embryos die at E9.5

\begin{tabular}{|l|c|c|c|c|}
\hline & ${\mathbf{a ~} \mathbf{T M 1}^{+/+}}^{+}$ & $\mathbf{a ~} \boldsymbol{T M 1}^{+/-}$ & $\mathbf{a}^{\mathbf{T M 1}^{-/-}}$ & Total \\
\hline E7.5 & 2 & 4 & 2 & 8 \\
\hline E8.5 & 8 & 18 & 10 & 36 \\
\hline E9.5 & 24 & 42 & 21 & 87 \\
\hline E10.5 & 6 & 17 & $2^{*}$ & 25 \\
\hline E11.5 & 4 & 14 & 0 & 18 \\
\hline E12.5 & 8 & 11 & 0 & 19 \\
\hline E15.5 & 4 & 8 & 0 & 12 \\
\hline Term & 101 & 177 & 0 & 278 \\
\hline
\end{tabular}

* These a TMI $1^{-/-}$embryos were severely stunted compared to their a $T M 1^{+/+}$and a $T M 1^{+/-}$littermates, signifying that they were in the process of resorption. 